\title{
Superradiant searches for dark photons in two stage atomic transitions
}

\author{
Amit Bhoonah $\odot,{ }^{1}$ Joseph Bramante, ${ }^{1,2}$ and Ningqiang Song $\oplus^{1,2}$ \\ ${ }^{1}$ The Arthur B. McDonald Canadian Astroparticle Physics Research Institute, Department of Physics, \\ Engineering Physics, and Astronomy, Queen's University, Kingston, Ontario K7L 2S8, Canada \\ ${ }^{2}$ Perimeter Institute for Theoretical Physics, Waterloo, Ontario N2L 2Y5, Canada
}

(Received 23 September 2019; accepted 11 March 2020; published 30 March 2020)

\begin{abstract}
We study a new mechanism to discover dark photon fields by resonantly triggering two-photon transitions in cold gas preparations. Using coherently prepared cold parahydrogen, the coupling sensitivity for sub-meV mass dark photon fields can be advanced by orders of magnitude with a modified lightshining-through-wall setup. We calculate the effect of a background dark photon field on the dipole moment and corresponding transition rate of cold parahydrogen pumped into its first vibrational excited state by counter-propagating laser beams. The nonlinear amplification of two-photon emission triggered by dark photons in a cold parahydrogen sample is numerically simulated to obtain the expected dark photon coupling sensitivity.
\end{abstract}

DOI: 10.1103/PhysRevD.101.055040

\section{INTRODUCTION}

The first glimmer of physics beyond the Standard Model may come from a new massive U(1) gauge boson weakly mixed with the photon, sometimes called a dark photon [1-3]. Dark photons are a predicted feature in supersymmetric theories, string theories, and hidden portal models of dark matter [4-9]. Many searches are underway to detect dark photons, produced either by a star [10-15], a laser [16-18], at colliders [19-22], or in the primordial Universe [23-27]. If they are produced in the early Universe, sub-MeV mass dark photons are a candidate model for dark matter [28-35].

Using two-stage atomic transitions, this paper proposes a new method to enhance the detection of dark photons produced by lasers shining through walls. This proposal involves a dark photon field produced in a laser cavity, and then passed through a sample of quasistable coherently excited atoms whose $E 1$ dipole transitions are parity forbidden. Under these conditions, during the brief time that the excited atoms are coherent, the diminutive field of the dark photon can resonantly trigger two-photon electronic transitions. As compared to traditional light-shining-throughwall experiments, we project a large gain in sensitivity to dark photons with $\mu \mathrm{eV}-\mathrm{meV}$ masses. These sensitivity gains appear within reach using preparations of parahydrogen $\left(\mathrm{pH}_{2}\right)$ coherently excited by counter-propagating nanosecond laser pulses [36,37].

Published by the American Physical Society under the terms of the Creative Commons Attribution 4.0 International license. Further distribution of this work must maintain attribution to the author(s) and the published article's title, journal citation, and DOI. Funded by SCOAP .
The use of two-stage superradiant atomic transitions for the production and detection of weakly coupled particles was proposed and studied extensively by Yoshimura et al. [36-44]. These authors along with Refs. [45-48] have studied how macroscopic quantities of coherently excited atoms may be employed to measure neutrino properties. The use of atomic transitions for the discovery of axion and dark photon dark matter was also recently considered in Refs. [49-53]. In contrast, the experiment we propose is sensitive to any $\mathrm{U}(1)$ vector bosons kinetically mixed with the Standard Model photon, whether or not dark matter is comprised of a dark photon.

Coherent superradiant emission by atomic systems was formalized by Dicke in Ref. [54]. However, the possibility that superradiance might be observed in macroscopic amalgams of materials has received increased attention in the last decade after being proposed as a method to measure certain neutrino properties $[39,40]$. Before proceeding further, we will develop some physical intuition about classic (a.k.a. Dicke) versus macro (a.k.a. Yoshimura) superradiance. A formal derivation can be found in Appendix A. Let us consider a group of atomic emitters with number density $n$ occupying a volume $V$, which have been prepared in excited states, such that each excited atomic emitter is indistinguishable from the next (we want them to have the same phase). Let us suppose that some atom in the volume $V$ deexcites and emits a photon with momentum $\mathbf{k}_{1}$. Then, if a single, isolated atom has a photon emission rate $\Gamma_{0}$ and for the moment we neglect superradiant effects (superradiant effects would indeed be negligible if the spacing between atomic emitters is much greater than the wavelength of photons emitted, $n^{-1 / 3} \gg k_{1}^{-1}$ ), the emission rate of photons from the volume $V$ follows trivially: $\Gamma_{\text {tot }}=n V \Gamma_{0}$. 
However, if the wavelength of the emitted photon is larger than the interatomic spacing, there will be a superradiant enhancement of the rate of photon emission. In fact, in the case that the emitted photon's wavelength is much greater than the volume itself $\left(k_{1}^{-3} \gg V\right)$ the total rate of photon emission will be $\Gamma_{\text {tot }}=n^{2} V^{2} \Gamma_{0}$, because of superradiance. This superradiant enhancement can be understood from basic quantum principles as follows. First, momentum conservation tells us that an emitting atom in its final state will have a momentum of size $\sim k_{1}$. The uncertainty principle tells us that the atom is only localized over a distance $\sim \frac{1}{k_{1}}$. Altogether, these imply that it is not possible to determine which atom in the volume $k_{1}^{-3}$ emitted the photon. But we know that quantum mechanics tells us that the probability for an event to occur is the squared sum of all ways for the event to occur, and if we cannot distinguish between atoms, then we must sum over all atoms in the coherence volume $k_{1}^{-3}$ and square that sum to obtain the probability for emission. From this we obtain an extra factor of $n V$ in our superradiantly enhanced emission rate. This is illustrated in Fig. 1.

In the preceding heuristic argument, it is crucial to realize that it is the final-state momentum and corresponding spatial uncertainty of the emitting atoms that determines the volume over which superradiant emission can occur. Therefore, a process that somehow reduces the final-state momentum of atomic emitters can potentially result in superradiant emission over volumes larger than the wavelength of emitted photons. Perhaps the simplest such process is two-photon emission. In two-photon emission, the final-state momentum of the emitting atom will be the sum of emitted momenta, $-\Delta \mathbf{k}=\mathbf{k}_{1}+\mathbf{k}_{2}$. For back-to-back two-photon emission where $\mathbf{k}_{1} \approx-\mathbf{k}_{2}$, the superradiant emission volume $\Delta k^{-3}$ can in principle be arbitrarily large; it is only limited by the difference in momentum of the emitted photons. This is illustrated in Fig. 1. In practice, the
Dicke Superradiance

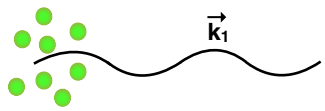

$-\Delta \overrightarrow{\mathbf{k}}=\overrightarrow{\mathbf{k}}_{1}$
Macro Superradiance
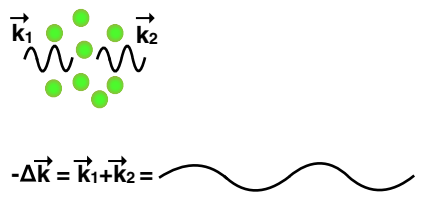

FIG. 1. Illustration of single-photon emission (a.k.a. Dicke superradiance) and two-photon emission (a.k.a. Yoshimura superradiance). Crucially, the volume for superradiant emission is determined by the final-state momentum $\Delta k$ of the atomic emitters. For classic Dicke superradiance this is just the momentum of the emitted photons, $\Delta k=\left|\mathbf{k}_{1}\right|$. For two photons emitted back to back, the final-state momentum can be tiny, $\Delta k \approx\left|\mathbf{k}_{1}-\mathbf{k}_{2}\right| \rightarrow 0$. Of course, superradiant emission will also depend on the linewidth of the lasers used to excite the atoms, material dephasing effects, and other factors; see Sec. II. dephasing of atoms, the related decoherence time of the atomic medium, and the linewidth of lasers used to excited the atoms will also limit the superradiant emission volume. It is interesting to note that much of the preceding logic about the cooperative emission of photons could be equally applied to cooperative absorption.

In this paper we show how two-photon superradiant emission by a large number of atoms can be used to detect dark photons. First, a sample of cold parahydrogen (or another suitable atomic target) is excited into a metastable state by back-to-back photons provided by counterpropagating lasers with frequency $\omega_{1}$. This excited state will preferentially decay through two-photon emission, in part because of the macroscopic superradiant enhancement detailed above. Next, dark photons produced in an adjacent cavity by a laser with frequency $\omega_{1}$ are passed through the excited parahydrogen sample. The dark photon field, through its mixing with the visible photon, would act as a "trigger laser," resonantly deexciting the excited parahydrogen through a two-photon transition. This altogether provides a new, very sensitive method to search for dark photons.

The rest of this paper proceeds as follows. In Sec. II we calculate coherence in two-stage electronic transitions, and study how much coherence is attained in cold preparations of parahydrogen excited by counter-propagating lasers. We find that the coherence necessary to begin realizing this proposal has been obtained in a number of experiments. The interaction of a dark photon with coherently excited two-stage atomic systems is derived in Sec. III. Enthusiastic readers may wish to skip to Sec. IV, which includes a schematic description of the experiment, along with its sensitivity to a kinetically mixed dark photon. Determining this dark photon sensitivity requires numerically integrating the dark and visible photon field equations in a background of coherently excited atoms. Conclusions are presented in Sec. V. Throughout, we use natural units where $\hbar=c=k_{B}=1$.

\section{COHERENCE IN TWO-STAGE ATOMIC TRANSITIONS}

Pulses from high-power lasers allow for the preparation of atoms in coherent excited states, from which they can be cooperatively deexcited. Before investigating how the weak electromagnetic field sourced by a dark photon can be detected by cooperatively deexciting coherently prepared atoms, it will be useful to examine under what conditions counter-propagating lasers excite highly coherent atoms in the first place. After deriving the coherence of atomic states excited by counter-propagating pairs of photons, we examine how laser power, atomic density, and temperature alter this coherence. The derivation given below can be found in many prior references $[55,56]$. Our aim here is to quantify the experimental capability-in terms of coherently excited atoms - that will be needed to detect dark photons. 


\section{A. Quasistable excited states}

We first consider an atomic system with ground state $|g\rangle$ and excited state $|e\rangle$. For the atomic systems we are interested in-for example, vibrational modes of parahydrogen, and electronic states of ytterbium or xenon [45]—both states $|g\rangle$ and $|e\rangle$ will have even parity, meaning that $E 1$ dipole transitions between the two states are forbidden. However, it will be possible to excite state $|g\rangle$ to state $|e\rangle$ through multiple $E 1$ dipole transitions, and similarly deexcite $|e\rangle$ to $|g\rangle$. So besides states $|g\rangle$ and $|e\rangle$, we consider intermediate states, $\left|j_{+}\right\rangle$and $\left|j_{-}\right\rangle$, where $+(-)$will indicate excitation into a positive (negative) angular momentum state by a circularly polarized photon. Figure 2 illustrates the basic setup. In physical realizations, there will be many $j$ states to transition through, such as, e.g., the $\ell=0,1,2,3 \ldots$.. electronic angular momentum states of hydrogen. Since by design these excited states will lie at energies beyond those provided by the input lasers, transitions through these states will be virtual. We define our atomic Hamiltonian as

$$
H=H_{0}+H_{I},
$$

where $H_{I}$ is the interaction Hamiltonian and $H_{0}$ is defined by $H_{0}|g\rangle=\omega_{g}|g\rangle, H_{0}|e\rangle=\omega_{e}|e\rangle, H_{0}\left|j_{ \pm}\right\rangle=\omega_{j}\left|j_{ \pm}\right\rangle$. With our states specified, we define the wave function for this simplified atomic system as

$$
\begin{aligned}
|\psi\rangle= & c_{g} e^{-i \omega_{g} t}|g\rangle+c_{e} e^{-i\left(\omega_{e}+\delta\right) t}|e\rangle+c_{j+} e^{-i \omega_{j} t}\left|j_{+}\right\rangle \\
& +c_{j-} e^{-i \omega_{j} t}\left|j_{-}\right\rangle .
\end{aligned}
$$

We have added a phase $\delta$ to account for detuning of the lasers; in other words, the laser beams exciting the atoms will be off resonance by a factor $\sim \delta$.

The laser-atom interaction Hamiltonian will depend on the orientation and quality of the impinging laser beams. Experimental setups similar to the one we are outlining (e.g., Ref. [37]) employ counter-propagating beams which

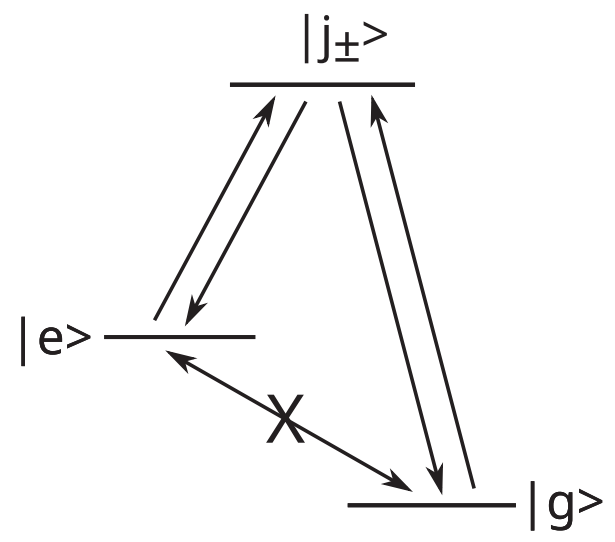

FIG. 2. Illustration of the energy levels of an atomic system, with ground state $|g\rangle$ and excited state $|e\rangle . E 1$ dipole transitions between $|g\rangle$ and $|e\rangle$ are forbidden; the two-step process of transitioning from $|g\rangle$ to $|e\rangle$ through a virtual state $\left|j_{ \pm}\right\rangle$is shown. have been circularly polarized. Therefore, we will consider two counter-propagating laser beams propagating along the $z$ direction with electric fields given as

$$
\begin{aligned}
\tilde{E}_{1}= & \frac{1}{2} E_{1}(z, t) \epsilon_{l} \exp \left\{-i \omega_{1}(t+z)\right\} \\
& +\frac{1}{2} E_{1}^{*}(z, t) \epsilon_{r} \exp \left\{i \omega_{1}(t+z)\right\}, \\
\tilde{E}_{2}= & \frac{1}{2} E_{2}(z, t) \epsilon_{l} \exp \left\{-i \omega_{2}(t-z)\right\} \\
& +\frac{1}{2} E_{2}^{*}(z, t) \epsilon_{r} \exp \left\{i \omega_{2}(t-z)\right\},
\end{aligned}
$$

where $\epsilon_{r}$ and $\epsilon_{l}$ are unit-normalized right- and left-handed polarization vectors for the laser beams. Then the laseratom interaction Hamiltonian is

$$
H_{I}=-\mathbf{d} \cdot\left(\tilde{E}_{1}+\tilde{E}_{2}\right)
$$

where $\mathbf{d}$ is the polarization of the atom. The actual dipole coupling and transition rate are experimental inputs in these formulas. Here we define the expectation value of the dipole transitions, with the assumption that both counterpropagating pump lasers will have left-handed circular polarization, using the convention that left-handedness is defined along the direction of the beam propagation. More explicitly, since $\tilde{E}_{2}$ is the electric field of a laser beam propagating in the $+\mathbf{z}$ direction,

$$
\begin{aligned}
& d_{j g} \equiv\left\langle j_{+}\left|-\mathbf{d} \cdot \epsilon_{r}\right| g\right\rangle=\left\langle j_{-}\left|-\mathbf{d} \cdot \epsilon_{l}\right| g\right\rangle, \\
& d_{j e} \equiv\left\langle j_{+}\left|-\mathbf{d} \cdot \epsilon_{r}\right| e\right\rangle=\left\langle j_{-}\left|-\mathbf{d} \cdot \epsilon_{l}\right| e\right\rangle,
\end{aligned}
$$

while for $\tilde{E}_{2}$

$$
\begin{aligned}
& \left\langle j_{+}\left|-\mathbf{d} \cdot \epsilon_{l}\right| g\right\rangle=\left\langle j_{-}\left|-\mathbf{d} \cdot \epsilon_{r}\right| g\right\rangle=0, \\
& \left\langle j_{+}\left|-\mathbf{d} \cdot \epsilon_{l}\right| e\right\rangle=\left\langle j_{-}\left|-\mathbf{d} \cdot \epsilon_{r}\right| e\right\rangle=0 .
\end{aligned}
$$

The same relations hold for $\tilde{E}_{1}$, except with $\epsilon_{l} \leftrightarrow \epsilon_{r}$, since $\tilde{E}_{1}$ is the electric field of the laser beam propagating in the $-\mathbf{z}$ direction. Finally, assuming that the two lasers will carry the same frequency (up to a detuning factor $\delta$ ), we define $\omega \equiv \omega_{1}=\omega_{2}=\omega_{e g} / 2$, and throughout this paper we use the convention that $\omega_{i k}$ for any states $i, k$ is defined as $\omega_{i k}=\omega_{i}-\omega_{k}$. Thus, we arrive at the following Schrödinger equations for this multistate atomic system:

$i \partial_{t} c_{j+}=\frac{1}{2}\left(d_{j g} c_{g} e^{i \omega_{j g} t}+d_{j e} c_{e} e^{i\left(\omega_{j e}-\delta\right) t}\right)\left(\bar{E}_{1} e^{-i \omega t}+\bar{E}_{2}^{*} e^{i \omega t}\right)$,

$i \partial_{t} c_{j-}=\frac{1}{2}\left(d_{j g} c_{g} e^{i \omega_{j g} t}+d_{j e} c_{e} e^{i\left(\omega_{j e}-\delta\right) t}\right)\left(\bar{E}_{1}^{*} e^{i \omega t}+\bar{E}_{2} e^{-i \omega t}\right)$, 


$$
\begin{aligned}
i \partial_{t} c_{g}= & \frac{1}{2} d_{g j} e^{-i \omega_{j g} t}\left[c_{j+}\left(\bar{E}_{1}^{*} e^{i \omega t}+\bar{E}_{2} e^{-i \omega t}\right)\right. \\
& \left.+c_{j-}\left(\bar{E}_{1} e^{-i \omega t}+\bar{E}_{2}^{*} e^{i \omega t}\right)\right], \\
i \partial_{t} c_{e}= & \frac{1}{2} d_{e j} e^{-i\left(\omega_{j e}-\delta\right) t}\left[c_{j+}\left(\bar{E}_{1}^{*} e^{i \omega t}+\bar{E}_{2} e^{-i \omega t}\right)\right. \\
& \left.+c_{j-}\left(\bar{E}_{1} e^{-i \omega t}+\bar{E}_{2}^{*} e^{i \omega t}\right)\right],
\end{aligned}
$$

where we have incorporated the spatial part of the electric fields into "barred" quantities, $\bar{E}_{1}=E_{1} e^{-i \omega z}$ and $\bar{E}_{2}=E_{2} e^{i \omega z}$. The sum over all intermediate states $j$ is implicit in Eqs. (8)-(11).

To find the time evolution of this system, we first integrate Eqs. (8) and (9) over $t$. We will be using the so-called Markov approximation, treating $c_{g}$ and $c_{e}$ as constants in the resulting integral. This standard approximation is justified, so long as we expect virtual transitions through $c_{j+}$ and $c_{j-}$ to be sufficiently rapid compared to changes in $c_{g}$ and $c_{e}$, which should be satisfied so long as the frequency of the $|g\rangle \rightarrow|e\rangle$ transition is substantially smaller than the frequency of higher-energy atomic states, $\omega_{e g} \ll \omega_{j e}, \omega_{j g}$. For example, in the case of $\mathrm{pH}_{2}$ the frequency of the first vibrational state, $\omega_{e g} \sim 0.5 \mathrm{eV}$, can be compared to the lowest-lying electronic excitations, $\omega_{j e}$ $\omega_{j g} \sim 10 \mathrm{eV}$, from which we conclude that the Markov approximation is justified. Using similar logic, we approximate the electric fields of the laser beams as being constant in this integral, since the laser frequency is also small compared to the transition frequencies to intermediate $j$ states. Setting the initial condition $c_{j_{ \pm}, 0}=0$, we find the time evolution of $c_{j+}$ and $c_{j-}$,

$$
\begin{aligned}
c_{j+}= & -\frac{1}{2}\left[d_{j g} c_{g} \frac{e^{i\left(\omega_{j g}-\omega\right) t}-1}{\omega_{j g}-\omega} \bar{E}_{1}+d_{j g} c_{g} \frac{e^{i\left(\omega_{j g}+\omega\right) t}-1}{\omega_{j g}+\omega} \bar{E}_{2}^{*}\right. \\
& \left.+d_{j e} c_{e} \frac{e^{i\left(\omega_{j e}-\delta-\omega\right) t}-1}{\omega_{j e}-\delta-\omega} \bar{E}_{1}+d_{j e} c_{e} \frac{e^{i\left(\omega_{j e}-\delta+\omega\right) t}-1}{\omega_{j e}-\delta+\omega} \bar{E}_{2}^{*}\right],
\end{aligned}
$$

$$
\begin{aligned}
c_{j-}= & -\frac{1}{2}\left[d_{j g} c_{g} \frac{e^{i\left(\omega_{j g}-\omega\right) t}-1}{\omega_{j g}-\omega} \bar{E}_{2}+d_{j g} c_{g} \frac{e^{i\left(\omega_{j g}+\omega\right) t}-1}{\omega_{j g}+\omega} \bar{E}_{1}^{*}\right. \\
& \left.+d_{j e} c_{e} \frac{e^{i\left(\omega_{j e}-\delta-\omega\right) t}-1}{\omega_{j e}-\delta-\omega} \bar{E}_{2}+d_{j e} c_{e} \frac{e^{i\left(\omega_{j e}-\delta+\omega\right) t}-1}{\omega_{j e}-\delta+\omega} \bar{E}_{1}^{*}\right] .
\end{aligned}
$$

Substituting these solutions for $c_{j+}$ and $c_{j-}$ into the Schrödinger equations for $c_{g}$ and $c_{e}$, we invoke the slowly varying envelope approximation, i.e., we assume that since the development of the electric fields around the atoms is slow compared to the frequencies of all transitions, all timedependent exponentials of the form $e^{i\left(\omega_{j e}-\delta+\omega\right) t} \approx e^{i\left(\omega_{j g}+\omega\right) t} \approx e^{i\left(\omega_{j e}-\delta-\omega\right) t} \approx e^{i\left(\omega_{j g}-\omega\right) t} \approx 0$

can be set to zero. With the slowly varying envelope approximation, the two-state system can be compactly expressed as

$$
i \partial_{t}\left(\begin{array}{c}
c_{e} \\
c_{g}
\end{array}\right)=H_{\mathrm{eff}}\left(\begin{array}{c}
c_{e} \\
c_{g}
\end{array}\right)
$$

with the effective Hamiltonian

$$
-H_{\mathrm{eff}}=\left(\begin{array}{cc}
\Omega_{e e} & \Omega_{e g} \\
\Omega_{g e} & \Omega_{e e}
\end{array}\right),
$$

where $\Omega_{g e}$ is the Rabi frequency of the system,

$$
\begin{aligned}
& \Omega_{e e}=\frac{a_{e e}}{4}\left(\left|\bar{E}_{1}\right|^{2}+\left|\bar{E}_{2}\right|^{2}\right), \\
& \Omega_{g g}=\frac{a_{g g}}{4}\left(\left|\bar{E}_{1}\right|^{2}+\left|\bar{E}_{2}\right|^{2}\right), \\
& \Omega_{e g}=\Omega_{g e}^{*}=\frac{a_{g e}}{2} \bar{E}_{1} \bar{E}_{2},
\end{aligned}
$$

and we have defined interstate dipole couplings as in Ref. [56],

$$
\begin{aligned}
& a_{e e}=\sum_{j}\left|d_{j e}\right|^{2}\left(\frac{1}{\omega_{j e}-\delta-\omega}+\frac{1}{\omega_{j e}-\delta+\omega}\right), \\
& a_{g g}=\sum_{j}\left|d_{j g}\right|^{2}\left(\frac{1}{\omega_{j g}-\omega}+\frac{1}{\omega_{j g}+\omega}\right), \\
& a_{e g}=a_{g e}^{*}=\sum_{j} \frac{d_{j e} d_{g j}}{\omega_{j e}-\delta+\omega} .
\end{aligned}
$$

Applying the density matrix of the atomic system

$$
\rho=\left(\begin{array}{ll}
|e\rangle\langle e| & |e\rangle\langle g| \\
|g\rangle\langle e| & |g\rangle\langle g|
\end{array}\right)=\left(\begin{array}{ll}
\rho_{e e} & \rho_{e g} \\
\rho_{g e} & \rho_{g g}
\end{array}\right)
$$

to the von Neumann equation $i \partial_{t} \rho=\left[H_{\text {eff }}, \rho\right]$ leads to the Maxwell-Bloch equations

$\partial_{t} \rho_{e e}=i\left(\Omega_{e g} \rho_{g e}-\Omega_{g e} \rho_{e g}\right)-\frac{\rho_{e e}}{T_{1}}$,

$\partial_{t} \rho_{g e}=i\left(\Omega_{g g}-\Omega_{e e}-\delta\right) \rho_{g e}+i \Omega_{g e}\left(\rho_{e e}-\rho_{g g}\right)-\frac{\rho_{g e}}{T_{2}}$,

$\partial_{t} \rho_{g g}=i\left(\Omega_{g e} \rho_{e g}-\Omega_{e g} \rho_{g e}\right)+\frac{\rho_{e e}}{T_{1}}$.

The final terms in Eqs. (24)-(26) have been added to account for spontaneous $|e\rangle \rightarrow|g\rangle$ transitions and the 
decoherence time of the mixed state. As such, $T_{1}$ is the excited-state lifetime and $T_{2}$ is the decoherence time.

To better quantify the coherence of this system, we define the Bloch vector $\mathbf{r}=\operatorname{Tr}(\sigma \rho)$ where $\sigma$ are the Pauli matrices. This implies

$$
\begin{aligned}
& r_{1}=\rho_{g e}+\rho_{e g}, \\
& r_{2}=i\left(\rho_{e g}-\rho_{g e}\right), \\
& r_{3}=\rho_{e e}-\rho_{g g} .
\end{aligned}
$$

By construction, $r_{1}$ and $r_{2}$ quantify the degree to which the atoms are coherently excited in the system, with maximum coherence attained when $r_{1}=1$ and $r_{3}=0$. The Bloch vector direction $r_{3}$ indicates the population difference between the excitation and the ground states. Note that $r_{1}, r_{2}$, and $r_{3}$ are all real numbers. Applying the Bloch vector basis to Eqs. (24)-(26), we obtain

$$
\begin{aligned}
\partial_{t} r_{1}= & {\left[-\frac{a_{g g}-a_{e e}}{4}\left(\left|\bar{E}_{1}\right|^{2}+\left|\bar{E}_{2}\right|^{2}\right)+\delta\right] r_{2} } \\
& +a_{e g} \operatorname{Im}\left(\bar{E}_{1} \bar{E}_{2}\right) r_{3}-\frac{r_{1}}{T_{2}}, \\
\partial_{t} r_{2}= & {\left[\frac{a_{g g}-a_{e e}}{4}\left(\left|E_{1}\right|^{2}+\left|E_{2}\right|^{2}\right)-\delta\right] r_{1} } \\
& +a_{e g} \operatorname{Re}\left(\bar{E}_{1} \bar{E}_{2}\right) r_{3}-\frac{r_{2}}{T_{2}}, \\
\partial_{t} r_{3}= & -a_{e g}\left[\operatorname{Im}\left(\bar{E}_{1} \bar{E}_{2}\right) r_{1}+\operatorname{Re}\left(\bar{E}_{1} \bar{E}_{2}\right) r_{2}\right]-\frac{1+r_{3}}{T_{1}},
\end{aligned}
$$

where we note that $a_{e g}$ is assumed to be real.

\section{B. Quantifying coherence in quasistable excited states}

Using the Bloch vector time evolution given by Eqs. (30)(32), we can now determine the degree and duration of coherence in cold atomic preparations excited by two counter-propagating lasers with electric fields $\tilde{E}_{1}$ and $\tilde{E}_{2}$. We consider an excited set of atoms with an expected spontaneous deexcitation time (not including superradiant enhancement) of $T_{1}$ and a decoherence time $T_{2}$. In the case of the first vibrationally excited state of $\mathrm{pH}_{2}$, the total lifetime has been observed to be $T_{1} \sim 10 \mu \mathrm{s}$ at $\sim 10 \mathrm{~K}$ temperatures [57-59], which will be appreciably longer than the decoherence time of the first $\mathrm{pH}_{2}$ vibrational excitation at these temperatures, where this decoherence time will be of order $\sim 1-100$ ns [60].

In more detail, the decoherence time $\left(T_{2}\right)$ of $\mathrm{pH}_{2}$ has been studied extensively for a variety of temperatures and densities $[37,40,44,60]$. In some regimes, it is accurate to use the mean interaction time of hydrogen atoms as an estimate of the decoherence time resulting from $\mathrm{pH}_{2}$ collisions at number density $n$,

$$
\begin{aligned}
t_{d c}= & \frac{1}{n_{H} \sigma_{H} \sqrt{2 T / m_{H}}} \\
\approx & 3 \mathrm{~ns}\left(\frac{3 \times 10^{19} \mathrm{~cm}^{-3}}{n}\right)\left(\frac{9 \times 10^{-17} \mathrm{~cm}^{2}}{\sigma_{H}}\right) \\
& \times\left(\frac{80 \mathrm{~K}}{T}\right)^{1 / 2}\left(\frac{m_{H}}{0.94 \mathrm{GeV}}\right)^{1 / 2},
\end{aligned}
$$

where this expression has approximated the velocity of $\mathrm{pH}_{2}$ using the temperature $(T)$ and mass $\left(m_{H}\right)$ of hydrogen, and the cross section using the Bohr radius, $\sigma_{H} \approx \pi r_{\text {Bohr }}^{2}$.

While Eq. (33) is remarkably close to the measured decoherence time for a sample of $\mathrm{pH}_{2}$ prepared at $T \sim 80 \mathrm{~K}$ and with a density $n \sim 3 \times 10^{19} \mathrm{~cm}^{-3}$, this approximation will break down for sufficiently cold and dense $\mathrm{pH}_{2}$, which will not behave like an ideal gas. In addition, we should note that the Raman linewidth, or the full width at half maximum of $\mathrm{pH}_{2}$ 's first vibrational emission line, is often used to determine the decoherence time. However, this linewidth also has a contribution from Doppler broadening of $\mathrm{pH}_{2}$,

$$
\Delta \nu_{\mathrm{dec}}^{(\text {Doppler })} \approx \omega_{0} \sqrt{\frac{T}{m_{H}}},
$$

where here $\omega_{0}$ is the first vibrational mode frequency. A total decoherence determination for the first vibrational mode of $\mathrm{pH}_{2}$, for temperatures ranging from $77-500 \mathrm{~K}$, was approximated by fitting the phenomenological formula [60]

$$
\Delta \nu_{\mathrm{dec}}=\frac{A}{n}+B n,
$$

where, for example, it was found that for $T=80 \mathrm{~K}$ the collisional term $A \approx 100 \mathrm{MHzcm}^{3}$, and the broadening term $B \approx 20 \mathrm{MHz} \mathrm{cm}^{3}$, which implies a $10 \mathrm{~ns}$ decoherence time for $n \sim 10^{19} \mathrm{~cm}^{-3}$, as previously noted.

Given the theoretical expectations and experimental results detailed above, it is safe to assume that $T_{2} \approx$ $10 \mathrm{~ns}$ is an achievable decoherence time for cold

TABLE I. A number of parahydrogen experiments with long decoherence times are listed for comparison, along with their temperatures and number densities. For $\mathrm{pH}_{2}$ Raman linewidth measurements $\Delta \nu_{\mathrm{dec}}$, the decoherence times are estimated as $T_{2} \sim \frac{1}{\Delta \nu_{\text {dec }}}$. In the case of Refs. [37,42], the decoherence time is an estimate using results from Ref. [60].

\begin{tabular}{lccc}
\hline \hline $\begin{array}{l}\mathrm{pH}_{2} \\
\text { Reference }\end{array}$ & $\begin{array}{c}\text { Density } \\
\left(\mathrm{cm}^{-3}\right)\end{array}$ & $\begin{array}{c}\text { Temperature } \\
(\mathrm{K})\end{array}$ & $\begin{array}{c}\text { Decoherence Time } \\
(\mathrm{ns})\end{array}$ \\
\hline$[60]$ & $10^{19}-10^{20}$ & $80-500$ & $\sim 10$ \\
{$[42]$} & $5.6 \times 10^{19}$ & 78 & $\sim 8(\mathrm{est})$ \\
{$[37]$} & $10^{19}-5 \times 10^{20}$ & 78 & $\sim 10(\mathrm{est})$ \\
{$[63]$} & $2.6 \times 10^{22}$ & 4.2 & $\gtrsim 140$ \\
\hline \hline
\end{tabular}



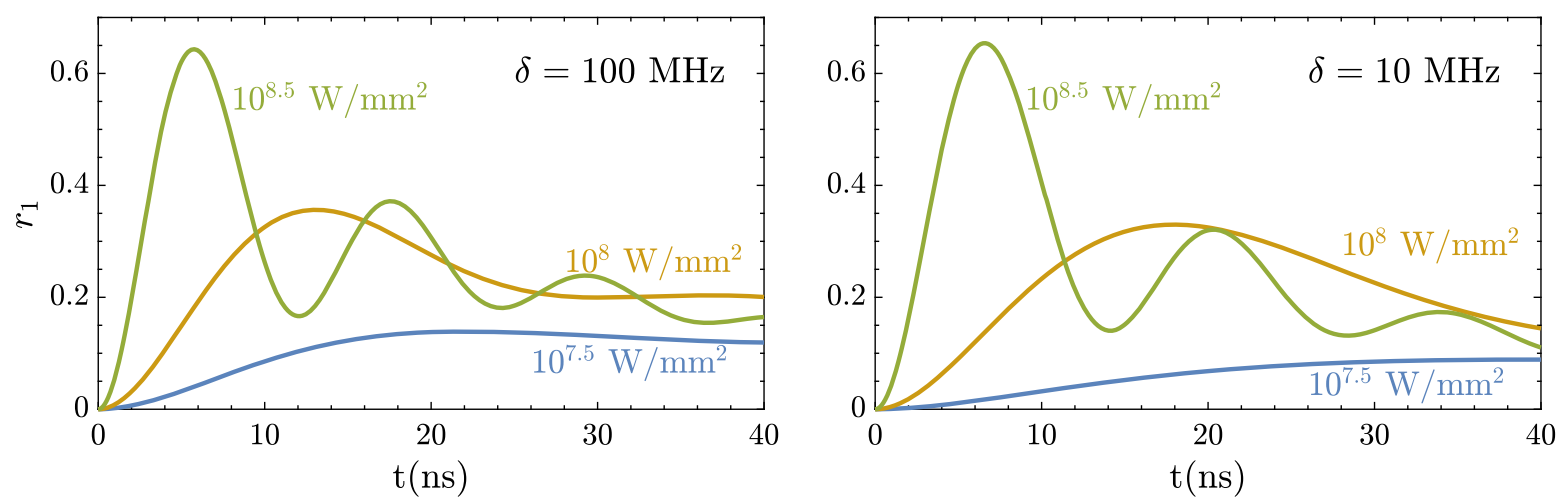

FIG. 3. The development of coherence in parahydrogen pumped by two counter-propagating lasers tuned to half the frequency of the first vibrational state of parahydrogen, $\omega=0.26 \mathrm{eV}$, with an assumed parahydrogen number density of $n=10^{21} \mathrm{~cm}^{-3}$. Results were obtained by solving Eqs. (30)-(32), where we take $a_{g g}=0.90 \times 10^{-24}, a_{e e}=0.87 \times 10^{-24}$, and $a_{e g}=0.0275 \times 10^{-24} \mathrm{~cm}^{3}[40]$. The intensity of the pump lasers is indicated. For comparison, we note that a coherence of $r_{1} \simeq 0.07$ has been achieved for parahydrogen at a density of $5 \times 10^{19} \mathrm{~cm}^{-3}$ using lasers less powerful than those assumed here [36]. However, the nanosecond-pulse gigawatt-power lasers required are commercially available [61]. (Indeed, even continuous gigawatt lasers as powerful as we require have been demonstrated in recent years [62].) The left panel assumes an experimental detuning $\delta=100 \mathrm{MHz}$, as achieved in recent counter-propagating pulsed laser experiments [37]. The right panel assumes $\delta=10 \mathrm{MHz}$, a linewidth that has been achieved in solid parahydrogen [63].

parahydrogen. In terms of the Bloch vector $r_{1}$, the largest coherence reported in a similar setup was $r_{1} \simeq 0.07$ for parahydrogen at a density $n \sim 5 \times 10^{19} \mathrm{~cm}^{-3}$ [36]. In the remainder of this paper, we will find that advancing the coupling sensitivity to dark photons (assuming a roughly $30 \mathrm{~cm}$ cylindrical chamber and $1 \mathrm{~cm}$ laser beam diameter) requires parahydrogen number densities closer to $n \sim 10^{21} \mathrm{~cm}^{-3}$. As noted in Fig. 3, a higher-power laser than that used in Ref. [36] is also required. In Fig. 3 we show how the coherence of $\mathrm{pH}_{2}$ can be expected to develop in time for $n \sim 10^{21} \mathrm{~cm}^{-3}$ by solving Eqs. (30)-(32), assuming a $\sim 10$ ns decoherence time and intrinsic detunings by experimental effects like Doppler broadening of both $\delta=10$ and $\delta=100 \mathrm{MHz}$. We will see that in this $\sim 10$ ns time frame a dark photon field applied to the cold atoms can greatly enhance the two-photon transition rate.

\section{DARK PHOTONS IN TWO-STAGE ATOMIC TRANSITIONS}

We have found that substantial coherence can be established in atoms excited by counter-propagating lasers, through a two-photon excitation process. Similarly, in the presence of a dark photon field, the rate for two-photon deexcitation can be resonantly enhanced. Suitably applied to coherently excited atoms, we will find that very weakly coupled dark photon fields can trigger two-photon transitions, during the $\sim 10 \mathrm{~ns}$ window of time that the atoms are coherently excited.

\section{A. Two-photon transitions with kinetic mixing}

We begin with the dark photon. The dark photon field is a new massive U(1) gauge field that kinetically mixes with the Standard Model photon. Its Lagrangian has the general form

$$
\begin{aligned}
\mathcal{L}= & -\frac{1}{4}\left(F_{\mu \nu} F^{\mu \nu}-2 \chi F_{\mu \nu} F^{\prime \mu \nu}+F_{\mu \nu}^{\prime} F^{\prime \mu \nu}\right) \\
& +\frac{m_{A^{\prime}}^{2}}{2} A_{\mu}^{\prime} A^{\prime \mu}-e J_{\mathrm{em}}^{\mu} A_{\mu},
\end{aligned}
$$

where $A^{\mu}$ and $A^{\prime \mu}$ are the four-vector potentials of the ordinary photon and dark photon field, and $F_{\mu \nu}$ and $F^{\prime \mu \nu}$ describe their field strength, respectively. Additionally, the dark photon is characterized by a mass $m_{A^{\prime}}$ and the kinetic mixing is suppressed by a constant $\chi$. Here $J_{\mathrm{em}}^{\mu}=\bar{\psi} \gamma^{\mu} \psi$ corresponds to the electromagnetic charged current with charged fermions $\psi$.

There is no direct coupling between the dark photon and charged fermions in Eq. (36). Rather, an effective interaction is introduced through kinetic mixing between the photon and dark photon, so long as $m_{A^{\prime}}>0$. Equivalently, one can diagonalize the kinetic mixing term by redefinition of the photon field $A_{\mu} \rightarrow A_{\mu}+\chi A_{\mu}^{\prime}$. To first order in $\chi$, we obtain the Lagrangian

$$
\begin{aligned}
\mathcal{L}= & -\frac{1}{4}\left(F_{\mu \nu} F^{\mu \nu}+F_{\mu \nu}^{\prime} F^{\prime \mu \nu}\right)+\frac{m_{A^{\prime}}^{2}}{2} A_{\mu}^{\prime} A^{\prime \mu} \\
& -e\left(A_{\mu}+\chi A_{\mu}^{\prime}\right) J_{\mathrm{em}}^{\mu} .
\end{aligned}
$$

To find the dark photon absorption and emission amplitudes in atomic transitions, it will be convenient to work with the effective Hamiltonian for electrons in the nonrelativistic limit in the presence of the dark photon field. Substituting the interaction terms in Eq. (37) into the Dirac Lagrangian,

$$
\mathcal{L}=i \bar{\psi} \gamma^{\mu} \partial_{\mu} \psi-m_{e} \bar{\psi} \psi-e\left(A_{\mu}+\chi A_{\mu}^{\prime}\right) J_{\mathrm{em}}^{\mu},
$$

we arrive at the Dirac equation for the electron 


$$
\left[i \not \supset-e\left(\not A+\chi \not^{\prime}\right)-m_{e}\right] \psi=0,
$$

where $m_{e}$ is the electron mass.

It will be convenient to work in the Dirac basis and divide the spinor into a dominant component $\psi_{d}$ and a subdominant component $\psi_{s}$, i.e., $\psi=\left(\psi_{d}, \psi_{s}\right)^{T}$. Separating out the time derivative from the Dirac equation, we find the Hamiltonian for the system

$$
i \partial_{t}\left(\begin{array}{c}
\psi_{d} \\
\psi_{s}
\end{array}\right)=H\left(\begin{array}{c}
\psi_{d} \\
\psi_{s}
\end{array}\right)
$$

where

$$
H=\left(\begin{array}{cc}
e\left(\Phi+\chi A_{0}^{\prime}\right)+m_{e} & -i \sigma \cdot \nabla-e \sigma \cdot\left(\mathbf{A}+\chi \mathbf{A}^{\prime}\right) \\
-i \sigma \cdot \nabla-e \sigma \cdot\left(\mathbf{A}+\chi \mathbf{A}^{\prime}\right) & e\left(\Phi+\chi A_{0}^{\prime}\right)-m_{e}
\end{array}\right) .
$$

Here $\sigma$ are the Pauli spin matrices and the electric potential $\Phi=A_{0}$.

The nonrelativistic Hamiltonian for this system is obtained by subtracting $m_{e}$ from both sides of Eq. (40), which yields

$$
H_{n r} \psi_{d}=-\left[i \sigma \cdot \nabla+e \sigma \cdot\left(\mathbf{A}+\chi \mathbf{A}^{\prime}\right)\right] \psi_{s}+e\left(\Phi+\chi A_{0}^{\prime}\right) \psi_{d},
$$

$$
\begin{aligned}
H_{n r} \psi_{s}= & -\left[i \sigma \cdot \nabla+e \sigma \cdot\left(\mathbf{A}+\chi \mathbf{A}^{\prime}\right)\right] \psi_{d}+e\left(\Phi+\chi A_{0}^{\prime}\right) \psi_{s} \\
& -2 m_{e} \psi_{s} .
\end{aligned}
$$

The subdominant component $\psi_{s}$ can be solved in the nonrelativistic limit where $\left|H_{n r}\right| \ll m_{e}$ and $\left|e\left(\Phi+A_{0}^{\prime}\right)\right| \ll m_{e}$. It is then substituted into Eq. (42) to obtain

$$
\begin{aligned}
H_{n r} & =H-m_{e} \\
& =\frac{1}{2 m_{e}}\left[i \sigma \cdot \nabla+e \sigma \cdot\left(\mathbf{A}+\chi \mathbf{A}^{\prime}\right)\right]^{2}+e\left(\Phi+\chi A_{0}^{\prime}\right),
\end{aligned}
$$

where this expression is valid in the nonrelativistic limit where Eq. (44) gives the effective Hamiltonian for an electron in the presence of electromagnetic and dark photon fields. Subtracting from it the standard QED Hamiltonian, we single out the components introduced by the new dark photon field,

$$
\begin{aligned}
H_{A^{\prime}}= & \frac{e \chi}{2 m_{e}}\left[i\left(\nabla \cdot \mathbf{A}^{\prime}+\mathbf{A}^{\prime} \cdot \nabla\right)\right]-\frac{e \chi}{2 m_{e}} \sigma \cdot\left(\nabla \times \mathbf{A}^{\prime}\right)+e \chi A_{0}^{\prime} \\
& +\frac{e^{2} \chi}{m_{e}} \mathbf{A} \cdot \mathbf{A}^{\prime}+\frac{e^{2} \chi^{2}}{2 m_{e}} \mathbf{A}^{\prime 2} .
\end{aligned}
$$

The first line of Eq. (45) reminds us of the standard QED Hamiltonian, with an additional gauge field. The $\frac{e^{2} \chi}{m_{e}} \mathbf{A} \cdot \mathbf{A}^{\prime}$ term arises from the expansion of the bracket in Eq. (44), meaning that even if the kinetic mixing is explicitly removed by a specific gauge choice in Eq. (37), dark photon and photon fields can still act on electrons in a collective way.

With the effective Hamiltonian in hand, we are now prepared to compute the transition amplitude from an initial atomic state $|i\rangle$ to a final state $|f\rangle$ with the absorption or emission of a dark photon. This transition has the general form

$$
\mathcal{M}=\left\langle f\left|H_{A^{\prime}}\right| i\right\rangle .
$$

We start with the first term in Eq. (45), which describes an $E 1$ (electric-dipole) type transition. Using the relation $\partial_{\mu} A^{\prime \mu}=0$, which can be readily obtained from the EulerLagrange equation (38) for the dark photon, we find

$$
\begin{aligned}
H_{A^{\prime}}^{E 1} & =i \frac{e \chi}{2 m_{e}}\left(\nabla \cdot \mathbf{A}^{\prime}+\mathbf{A}^{\prime} \cdot \nabla\right) \\
& =-\frac{e \chi}{2 m_{e}}\left(i \partial_{t} A_{0}^{\prime}+2 \mathbf{A}^{\prime} \cdot \mathbf{p}_{e}\right),
\end{aligned}
$$

where $\mathbf{p}_{e}$ is the momentum operator for the electron. Using the relation $\mathbf{p}_{e}=-i m_{e}\left[\mathbf{r}, H_{0}\right]$, where $H_{0}=p_{e}^{2} / 2 m_{e}$ is the unperturbed atomic Hamiltonian, we obtain

$$
\mathcal{M}^{E 1}=-\frac{e \chi}{2 m_{e}}\left\langle f\left|\partial_{t} A_{0}^{\prime}\right| i\right\rangle+i \omega_{i f} e \chi\left\langle f\left|\mathbf{A}^{\prime} \cdot \mathbf{r}\right| i\right\rangle,
$$

where again we note that $\omega_{i k} \equiv \omega_{i}-\omega_{k}$ is the energy difference between the initial and final atomic states. The first term in Eq. (48) is suppressed by a factor $\sim \omega / m_{e}$ and is therefore negligible compared to the second term. Hence, we drop this first term for simplicity.

To evaluate the second term, we define the vector component of the dark photon field as $\mathbf{A}^{\prime}=$ $\left|\mathbf{A}^{\prime}\right| \epsilon^{\prime} \exp (i \omega t-i \mathbf{k} \cdot \mathbf{r})$, which will have energy $\omega=\omega_{i f}$. Because we will be considering dipole moments substantially smaller than the wavelength of the applied laser (or the wavelength of the dark photon), the dipole approximation $\exp (-i \mathbf{k} \cdot \mathbf{r}) \simeq 1$ applies. With this approximation,

$$
\mathcal{M}^{E 1} \simeq i e \chi \omega_{i f}\left|\mathbf{A}^{\prime}\right|\langle f|\epsilon \cdot \mathbf{d}| i\rangle,
$$

where the $\mathbf{d}=e \mathbf{r}$ is the dipole operator. Following standard electromagnetic conventions, we define the dark electric field as

$$
\mathbf{E}^{\prime}=-\nabla V^{\prime}-\partial_{t} \mathbf{A}^{\prime}
$$

where

$$
V^{\prime}(\mathbf{r}, t)=\frac{i}{\omega} \nabla \cdot \mathbf{A}^{\prime}
$$

Assuming $\left|A^{\prime}\right|$ varies slowly in space and time, we obtain

$$
\mathbf{E}^{\prime}=\frac{i}{\omega}\left[\left(\mathbf{k} \cdot \mathbf{A}^{\prime}\right) \mathbf{k}-\omega^{2} \mathbf{A}^{\prime}\right]
$$


We decompose the dark electric field into a transverse component $\mathbf{E}_{T}^{\prime}$ and longitudinal component $\mathbf{E}_{L}^{\prime}$,

$$
\begin{aligned}
& \mathbf{E}_{T}^{\prime}=-i \omega \mathbf{A}_{T}^{\prime}, \\
& \mathbf{E}_{L}^{\prime}=-i \frac{m_{A^{\prime}}^{2}}{\omega} \mathbf{A}_{L}^{\prime} .
\end{aligned}
$$

If $\left|\mathbf{A}_{T}^{\prime}\right| \simeq\left|\mathbf{A}_{L}^{\prime}\right|$, we have $\left|\mathbf{E}_{L}^{\prime}\right| /\left|\mathbf{E}_{T}^{\prime}\right| \simeq m_{A^{\prime}}^{2} / \omega^{2}$. Note that our proposed experiment is only sensitive to dark photons with sub-meV masses, since this is a necessary condition for coherent excitation of two-stage atomic transitions in the target sample (see Sec. IV). While the dark photon masses will be $m_{A^{\prime}} \lesssim \mathrm{meV}$, the transition energy $\omega \sim \mathrm{eV}$, and therefore we expect the effect of the longitudinal component of the dark electric field to be subdominant since $\left|\mathbf{E}_{L}^{\prime}\right| /\left|\mathbf{E}_{T}^{\prime}\right| \simeq m_{A^{\prime}}^{2} / \omega^{2}$. Therefore, we only focus on the transverse component when computing the transition amplitude,

$$
\mathcal{M}^{E 1} \simeq-\chi\left\langle f\left|\mathbf{d} \cdot \mathbf{E}_{\mathbf{T}}^{\prime}\right| i\right\rangle .
$$

We could also evaluate the transition amplitude induced by other terms in Eq. (45). However, we note that the second term is characterized by $M 1$ (magnetic-dipole) type transition which is suppressed by $1 / m_{e}$ compared to $\mathcal{M}^{E 1}$. The third term vanishes in the leading-order expansion of $\exp (-i \mathbf{k} \cdot \mathbf{r})$. The fourth and last terms are also suppressed by $1 / m_{e}$, and the last term is further suppressed by $\chi$, and so we also drop these terms.

\section{B. Dark-photon-induced superradiance}

Now that we have obtained the dark photon dipole transition amplitude, we are ready to study dark-photoninduced superradiance. We will focus on the transition between the excitation state $|e\rangle$ and ground state $|g\rangle$ of a $\mathrm{pH}_{2}$ target. As previously noted, $|g\rangle$ and $|e\rangle$ will denote the zeroth and first vibrational state for $\mathrm{pH}_{2}$, where both of these have $J=0$. Since $|e\rangle$ and $|g\rangle$ share the same parity, an $E 1$ dipole transition is forbidden, but the transition between them can take place via two $E 1$ transitions by transitioning through an intermediate virtual state $|j\rangle$. Hence, we will compute $E 1 \times E 1$ transitions for which two particles are emitted, as shown in Figs. 4(a) and 4(b). As we mentioned in the previous section, the interaction between the dark photon and electron allows for this $E 1 \times E 1$ transition to occur via the emission of a dark photon and Standard Model photon $|e\rangle \rightarrow|g\rangle+\gamma^{\prime}+\gamma$, along with the standard two-photon emission process $|e\rangle \rightarrow|g\rangle+\gamma+\gamma$, illustrated in Figs. 4(a) and 4(b). These two processes will reinforce each other in a coherently excited atomic medium, since the emission of a dark photon can trigger and amplify the twophoton emission process, and vice versa. To demonstrate this mutual reinforcement, we shall derive the evolution

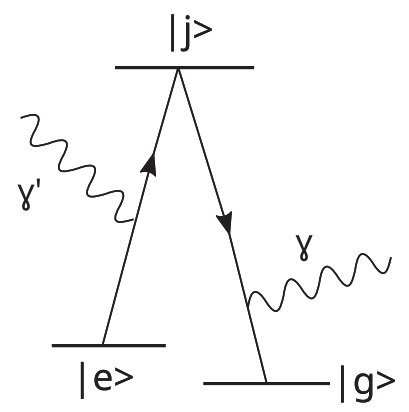

(a) $|e\rangle \rightarrow|g\rangle+\gamma^{\prime}+\gamma$

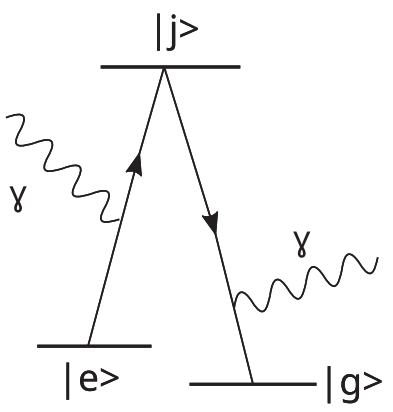

(b) $|e\rangle \rightarrow|g\rangle+\gamma+\gamma$
FIG. 4. Illustration of the two deexcitation processes. Panel (a) The transition from $|e\rangle$ to $|g\rangle$ with the emission of a photon and a dark photon. Panel (b) The transition from $|e\rangle$ to $|g\rangle$ with the emission of two photons.

equations of the dark photon and photon fields during deexcitation.

\section{Maxwell-Bloch equations}

First, we reformulate the Maxwell-Bloch equations as they were derived in Sec. II, now including the dark photon's effect on the electric dipole. As before, we denote the spin $m_{J}= \pm 1$ states as $\left|j_{ \pm}\right\rangle$. In addition to the two photon fields $E_{1}$ and $E_{2}$, we define a dark photon $E^{\prime}$ propagating in the positive $z$ direction,

$$
\tilde{E}^{\prime}=\frac{1}{2} E^{\prime}(z, t) \epsilon^{\prime} \exp \left\{-i \omega^{\prime} t+i k z\right\}+\text { c.c. }
$$

Because we are only considering the transverse component of the dark photon field, we take $\epsilon^{\prime}=\epsilon_{T}^{\prime}$. We expect that to good approximation $\omega_{1}=\omega_{2}=\omega^{\prime}=\omega=\omega_{e g} / 2$, since this is already required for coherence of the excited atomic state. We again write the $\mathrm{pH}_{2}$ wave function as the superposition of atomic states,

$$
\begin{aligned}
|\psi\rangle= & c_{g} e^{-i \omega_{g} t}|g\rangle+c_{e} e^{-i\left(\omega_{e}+\delta\right) t}|e\rangle+c_{j+} e^{-i \omega_{j} t}\left|j_{+}\right\rangle \\
& +c_{j-} e^{-i \omega_{j} t}\left|j_{-}\right\rangle .
\end{aligned}
$$

For the sake of simplicity, we will keep $\delta$ in the derivation, but set $\delta=0$ in the numerical simulations, which amounts to assuming that the atoms, lasers, and dark photon field are in phase over the target volume for time scales shorter than the decoherence time, $T_{2} \sim 10 \mathrm{~ns}$. For a full discussion of the physical requirements for $\sim 10 \mathrm{~ns}$ decoherence times, and the loss of coherence as $\delta$ is increased, see Sec. II. For discussions of the detuning effect on the output photon flux and the experimental sensitivity, see Appendix C. The full interaction Hamiltonian is then

$$
H_{I}=-\mathbf{d} \cdot\left(\tilde{E}_{1}+\tilde{E}_{2}+\chi \tilde{E}^{\prime}\right) .
$$

The Schrödinger equations will now include terms proportional to the dark photon field, 


$$
\begin{aligned}
i \partial_{t} c_{j+}= & \frac{1}{2}\left(d_{j g} c_{g} e^{i \omega_{j g} t}+d_{j e} c_{e} e^{i\left(\omega_{j e}-\delta\right) t}\right)\left(\bar{E}_{1} e^{-i \omega t}+\bar{E}_{2}^{*} e^{i \omega t}\right)+\frac{\chi}{2}\left(d_{j g}^{\prime} c_{g} e^{i \omega_{j g} t}+d_{j e}^{\prime} c_{e} e^{i\left(\omega_{j e}-\delta\right) t}\right)\left(\bar{E}^{\prime} e^{-i \omega t}+\bar{E}^{\prime *} e^{i \omega t}\right), \\
i \partial_{t} c_{j-}= & \frac{1}{2}\left(d_{j g} c_{g} e^{i \omega_{j g} t}+d_{j e} c_{e} e^{i\left(\omega_{j e}-\delta\right) t}\right)\left(\bar{E}_{1}^{*} e^{i \omega t}+\bar{E}_{2} e^{-i \omega t}\right)+\frac{\chi}{2}\left(d_{j g}^{\prime} c_{g} e^{i \omega_{j g} t}+d_{j e}^{\prime} c_{e} e^{i\left(\omega_{j e}-\delta\right) t}\right)\left(\bar{E}^{\prime *} e^{i \omega t}+\bar{E}^{\prime} e^{-i \omega t}\right), \\
i \partial_{t} c_{g}= & \frac{1}{2} d_{g j} e^{-i \omega_{j g} t}\left[c_{j+}\left(\bar{E}_{1}^{*} e^{i \omega t}+\bar{E}_{2} e^{-i \omega t}\right)+c_{j-}\left(\bar{E}_{1} e^{-i \omega t}+\bar{E}_{2}^{*} e^{i \omega t}\right)\right] \\
& +\frac{\chi}{2} d_{g j}^{\prime} e^{-i \omega_{j g} t}\left[c_{j+}\left(\bar{E}^{\prime *} e^{i \omega t}+\bar{E}^{\prime} e^{-i \omega t}\right)+c_{j-}\left(\bar{E}^{\prime} e^{-i \omega t}+\bar{E}^{\prime *} e^{i \omega t}\right)\right], \\
i \partial_{t} c_{e}= & \frac{1}{2} d_{e j} e^{-i\left(\omega_{j e}-\delta\right) t}\left[c_{j+}\left(\bar{E}_{1}^{*} e^{i \omega t}+\bar{E}_{2} e^{-i \omega t}\right)+c_{j-}\left(\bar{E}_{1} e^{-i \omega t}+\bar{E}_{2}^{*} e^{i \omega t}\right)\right] \\
& +\frac{\chi}{2} d_{e j}^{\prime} e^{-i\left(\omega_{j e}-\delta\right) t}\left[c_{j+}\left(\bar{E}^{\prime *} e^{i \omega t}+\bar{E}^{\prime} e^{-i \omega t}\right)+c_{j-}\left(\bar{E}^{\prime} e^{-i \omega t}+\bar{E}^{\prime *} e^{i \omega t}\right)\right],
\end{aligned}
$$

where (as in Sec. II) we absorb spatial dependence into the overbarred fields $\bar{E}_{1}=E_{1} e^{-i \omega z}, \bar{E}_{2}=E_{2} e^{i \omega z}$, and $\bar{E}^{\prime}=E^{\prime} e^{i k z}$. Note also that we have left implicit the sum over all intermediate states $j$ in Eqs. (61) and (62). Integrating Eqs. (59) and (60) over $t$, using the Markovian approximation, and imposing the initial condition $c_{j_{ \pm}, 0}=0$, we obtain

$$
\begin{aligned}
c_{j+}= & -\frac{1}{2} \sum_{s=g, e}\left[\frac{c_{s}}{\omega_{j s}-\omega-\Delta_{s e} \delta}\left(d_{j s} \bar{E}_{1}+\chi d_{j s}^{\prime} \bar{E}^{\prime}\right)\left(e^{i\left(\omega_{j s}-\omega-\Delta_{s e} \delta\right) t}-1\right)\right. \\
& \left.+\frac{c_{s}}{\omega_{j s}+\omega-\Delta_{s e} \delta}\left(d_{j s} \bar{E}_{2}^{*}+\chi d_{j s}^{\prime} \bar{E}^{\prime *}\right)\left(e^{i\left(\omega_{j s}+\omega-\Delta_{s e} \delta\right) t}-1\right)\right], \\
c_{j-}= & -\frac{1}{2} \sum_{s=g, e}\left[\frac{c_{s}}{\omega_{j s}-\omega-\Delta_{s e} \delta}\left(d_{j s} \bar{E}_{2}+\chi d_{j s}^{\prime} \bar{E}^{\prime}\right)\left(e^{i\left(\omega_{j s}-\omega-\Delta_{s e} \delta\right) t}-1\right)\right. \\
& \left.+\frac{c_{s}}{\omega_{j s}+\omega-\Delta_{s e} \delta}\left(d_{j s} \bar{E}_{1}^{*}+\chi d_{j s}^{\prime} \bar{E}^{\prime *}\right)\left(e^{i\left(\omega_{j s}+\omega\right) t-\Delta_{s e} \delta}-1\right)\right],
\end{aligned}
$$

where $\Delta_{s e}=0$ if $s=g$ and $\Delta_{s e}=1$ if $s=e$. Inserting Eqs. (63) and (64) into Eqs. (61) and (62) and using the slowly varying envelope approximation, we obtain the equation for the two-state system in the presence of a dark photon,

$$
i \partial_{t}\left(\begin{array}{c}
c_{e} \\
c_{g}
\end{array}\right)=-\left(\begin{array}{ll}
\Omega_{e e} & \Omega_{e g} \\
\Omega_{g e} & \Omega_{e e}
\end{array}\right)\left(\begin{array}{c}
c_{e} \\
c_{g}
\end{array}\right),
$$

where the $2 \times 2$ matrix is the effective Hamiltonian $\left(H_{\mathrm{eff}}\right)$, and its components are

$$
\begin{aligned}
& \Omega_{e e}=\frac{a_{e e}}{4}\left(\left|\bar{E}_{1}+\chi \eta \bar{E}^{\prime}\right|^{2}+\left|\bar{E}_{2}+\chi \eta \bar{E}^{\prime}\right|^{2}\right), \\
& \Omega_{g g}=\frac{a_{g g}}{4}\left(\left|\bar{E}_{1}+\chi \eta \bar{E}^{\prime}\right|^{2}+\left|\bar{E}_{2}+\chi \eta \bar{E}^{\prime}\right|^{2}\right), \\
& \Omega_{e g}=\Omega_{g e}^{*}=\frac{a_{g e}}{2}\left(\bar{E}_{1}+\chi \eta \bar{E}^{\prime}\right)\left(\bar{E}_{2}+\chi \eta \bar{E}^{\prime}\right),
\end{aligned}
$$

where we have defined the dipole couplings $a_{e e}, a_{g g}$, and $a_{g e}$ as before. In contrast to Sec. II, we now also define

$$
\eta \equiv \frac{d_{j g}^{\prime}}{d_{j g}}=\frac{d_{j e}^{\prime}}{d_{j e}},
$$

which quantifies the relative phase between the polarization of the photon field and the dark photon field.

As before, we introduce the density matrix and add relaxation terms to obtain the Maxwell-Bloch equations,

$\partial_{t} \rho_{e e}=i\left(\Omega_{e g} \rho_{g e}-\Omega_{g e} \rho_{e g}\right)-\frac{\rho_{e e}}{T_{1}}$,

$\partial_{t} \rho_{g e}=i\left(\Omega_{g g}-\Omega_{e e}-\delta\right) \rho_{g e}+i \Omega_{g e}\left(\rho_{e e}-\rho_{g g}\right)-\frac{\rho_{g e}}{T_{2}}$,

where $T_{1}$ and $T_{2}$ are the relaxation and decoherence time, respectively.

We can expand Eq. (68) to make manifest oscillations in $\Omega_{e g}$,

$\Omega_{e g}=\frac{a_{e g}}{2}\left[\left(E_{1} E_{2}+\chi \eta E_{1} E^{\prime}\right)+\chi \eta\left(E^{\prime} E_{2}+\chi \eta E^{\prime 2}\right) e^{2 i \omega z}\right]$, 
where we assume that $\omega \simeq k$. From Eq. (71) we also need to decompose $\rho_{g e}$ correspondingly,

$$
\rho_{g e}=\rho_{g e}^{0}+\rho_{g e}^{-} e^{-2 i \omega z} .
$$

We note that $\rho_{g e}^{-}$only comes from the atomic excitation due to the absorption of $E_{2}$ and $E^{\prime}$ or two dark photons, and the coherence developed in these processes is small. Thus, to leading order we can drop the second term in Eq. (73) and assume no spatial phase in $\rho_{g e}$.

\section{Field equations}

The Bloch equations we have derived in the previous section show the evolution of the population of the ground state and excitation state in the presence of electric and dark electric fields. Now we would like to see how these fields evolve as the population changes. It is straightforward to obtain from Eq. (37) the field equations

$$
\begin{aligned}
\left(\partial_{t}^{2}-\partial_{z}^{2}\right) A^{\mu} & =e J_{\mathrm{em}}^{\mu}, \\
\left(\partial_{t}^{2}-\partial_{z}^{2}+m_{A^{\prime}}^{2}\right) A^{\prime \mu} & =e \chi J_{\mathrm{em}}^{\mu} .
\end{aligned}
$$

There is no free electric charge in the target and $\mathbf{J}_{\mathrm{em}}$ can be identified as the polarization current density determined by the polarization field

$$
e \mathbf{J}_{\mathrm{em}}=n \frac{\partial \tilde{P}}{\partial t},
$$

where $n$ is the number density of $\mathrm{pH}_{2}$. We recall the definition of $E^{\prime}$ in Eq. (50) and take the time derivative of both sides of Eqs. (74) and (75) to obtain

$$
\begin{aligned}
\left(\partial_{t}^{2}-\partial_{z}^{2}\right) \tilde{E}_{i} & =-n \partial_{t}^{2} \tilde{P}_{i}, \\
\left(\partial_{t}^{2}-\partial_{z}^{2}+m_{A^{\prime}}^{2}\right) \tilde{E}^{\prime} & =-\chi n \partial_{t}^{2} \tilde{P}^{\prime},
\end{aligned}
$$

where $i=1,2$ represent different electric fields. The polarization field arises from the dipole moment in the atomic transition, where

$$
\tilde{P}=\langle\psi|\mathbf{d}| \psi\rangle .
$$

Note that $\tilde{E}_{1}$ and $\tilde{E}_{2}$ propagate in opposite directions with opposite spin angular momenta, as the microscopic polarization that sources these fields is also different. Accounting for the conservation of angular momentum, we have

$$
\begin{aligned}
& -\tilde{P}_{1}=\sum_{s=g, e}\left(d_{s j} c_{s}^{*} c_{j+} e^{-i\left(\omega_{j s}-\omega-\Delta_{s e} \delta\right) t}+d_{j s} c_{j-}^{*} c_{s} e^{i\left(\omega_{j s}-\omega-\Delta_{s e} \delta\right) t}\right) \epsilon_{l}+\text { c.c., } \\
& -\tilde{P}_{2}=\sum_{s=g, e}\left(d_{s j} c_{s}^{*} c_{j+} e^{-i\left(\omega_{j s}-\omega-\Delta_{s e} \delta\right) t}+d_{j s} c_{j-}^{*} c_{s} e^{i\left(\omega_{j s}-\omega-\Delta_{s e} \delta\right) t}\right) \epsilon_{r}+\text { c.c. }, \\
& -\tilde{P}^{\prime}=\sum_{s=g, e}\left[d_{s j}^{\prime} c_{s}^{*}\left(c_{j+}+c_{j-}\right) e^{-i\left(\omega_{j s}-\omega-\Delta_{s e} \delta\right) t}+d_{j s}^{\prime} c_{s}\left(c_{j+}^{*}+c_{j-}^{*}\right) e^{i\left(\omega_{j s}-\omega-\Delta_{s e} \delta\right) t}\right] \epsilon^{\prime}+\text { c.c. }
\end{aligned}
$$

We work in the limit where $m_{A^{\prime}} \ll \omega$, so approximately $\omega \simeq k$. We can substitute $c_{j \pm}$ as given in Eqs. (63) and (64) into Eqs. (80) to (82) and keep only the terms containing $e^{ \pm i \omega t}$ [to match the left-hand sides of Eqs. (77) and (78)] to obtain

$$
\begin{aligned}
2 \tilde{P}_{1(2)}= & \left\{\left[\left(a_{e e} \rho_{e e}+a_{g g} \rho_{g g}\right) E_{1}+2 a_{e g} \rho_{g e}^{*}\left(E_{2}^{*}+\chi \eta E^{\prime *}\right)\right] e^{-i \omega(t+z)}+\left[\left(a_{e e} \rho_{e e}+a_{g g} \rho_{g g}\right)\left(E_{2}^{*}+\chi \eta E^{\prime *}\right)+2 a_{e g}^{*} \rho_{g e} E_{1}\right] e^{i \omega(t-z)}\right. \\
& \left.+\left[\left(a_{e e} \rho_{e e}+a_{g g} \rho_{g g}\right) e^{-i \omega(t-z)}+2 a_{e g}^{*} \rho_{g e} e^{i \omega(t+z)}\right] \chi \eta E^{\prime}\right\} \epsilon_{l(r)}+\text { c.c. }, \\
2 \tilde{P}^{\prime}=\{ & \left\{\left(a_{e e} \rho_{e e}+a_{g g} \rho_{g g}\right) E_{1}+2 a_{e g} \rho_{g e}^{*}\left(E_{2}^{*}+2 \chi \eta E^{\prime *}\right)\right] e^{-i \omega(t+z)}+\left[\left(a_{e e} \rho_{e e}+a_{g g} \rho_{g g}\right)\left(2 \chi \eta E^{\prime}+E_{2}\right)+2 a_{e g} \rho_{g e} E_{1}^{*}\right] e^{-i \omega(t-z)} \\
+ & {\left.\left[\left(a_{e e} \rho_{e e}+a_{g g} \rho_{g g}\right) E_{1}^{*}+2 a_{e g}^{*} \rho_{g e}\left(E_{2}+2 \chi \eta E^{\prime}\right)\right] e^{i \omega(t+z)}+\left[\left(a_{e e} \rho_{e e}+a_{g g} \rho_{g g}\right)\left(2 \chi \eta E^{\prime *}+E_{2}^{*}\right)+2 a_{e g}^{*} \rho_{g e} E_{1}\right] e^{i \omega(t-z)}\right\} \epsilon^{\prime} } \\
+ & \text { c.c. }
\end{aligned}
$$

By matching the oscillation phases of the electric fields and the microscopic polarization and using the slowly varying envelope approximation, we arrive at the field equations for $E_{1}, E_{2}$, and $E^{\prime}$

$$
\begin{aligned}
& \left(\partial_{t}-\partial_{z}\right) E_{1}=\frac{i \omega n}{2}\left[\left(a_{e e} \rho_{e e}+a_{g g} \rho_{g g}\right) E_{1}+2 a_{e g} \rho_{g e}^{*}\left(E_{2}^{*}+\chi \eta E^{\prime *}\right)\right], \\
& \left(\partial_{t}+\partial_{z}\right) E_{2}=\frac{i \omega n}{2}\left[\left(a_{e e} \rho_{e e}+a_{g g} \rho_{g g}\right)\left(E_{2}+\chi \eta E^{\prime}\right)+2 a_{e g} \rho_{g e}^{*} E_{1}^{*}\right], \\
& \left(\partial_{t}+\partial_{z}\right) E^{\prime}=\frac{i \omega^{2} n}{\omega+k}\left[\left(a_{e e} \rho_{e e}+a_{g g} \rho_{g g}\right)\left(2 \chi^{2} \eta E^{\prime}+\chi E_{2}\right)+2 a_{e g} \rho_{g e}^{*} \chi E_{1}^{*}\right] .
\end{aligned}
$$


The first terms on the right-hand sides of these equations, which are proportional to $a_{e e}$ and $a_{g g}$, do not affect the transition from excited to ground states, but rather describe absorption and reemission of photons or dark photons propagating in the medium. More importantly, the second terms on the right-hand sides of the above equations, proportional to $a_{e g}$, describe the production of electromagnetic fields via excited to ground state transitions of the atoms. Altogether, $E_{1}$ can be amplified by seed $E_{2}$ and $E^{\prime}$ fields, and correspondingly, $E_{2}$ and $E^{\prime}$ are amplified by the $E_{1}$ field through transitions. For our purposes, we are most interested in the fact that $E^{\prime}$ will amplify $E_{1}$ and $E_{2}$ in these equations, which forms the basis of our dark photon detection proposal.

\section{Bloch vector}

Defining the Bloch vector as in Sec. II, from Eqs. (70) and (71) we obtain

$$
\begin{aligned}
\partial_{t} r_{1}= & {\left[-\frac{a_{g g}-a_{e e}}{4}\left(\left|\bar{E}_{1}^{\prime}\right|^{2}+\left|\bar{E}_{2}^{\prime}\right|^{2}\right)+\delta\right] r_{2} } \\
& +a_{e g} \operatorname{Im}\left(\bar{E}_{1}^{\prime} \bar{E}_{2}^{\prime}\right) r_{3}-\frac{r_{1}}{T_{2}}, \\
\partial_{t} r_{2}= & {\left[\frac{a_{g g}-a_{e e}}{4}\left(\left|E_{1}^{\prime}\right|^{2}+\left|E_{2}^{\prime}\right|^{2}\right)-\delta\right] r_{1} } \\
& +a_{e g} \operatorname{Re}\left(\bar{E}_{1}^{\prime} \bar{E}_{2}^{\prime}\right) r_{3}-\frac{r_{2}}{T_{2}}, \\
\partial_{t} r_{3}= & -a_{e g}\left[\operatorname{Im}\left(\bar{E}_{1}^{\prime} \bar{E}_{2}^{\prime}\right) r_{1}+\operatorname{Re}\left(\bar{E}_{1}^{\prime} \bar{E}_{2}^{\prime}\right) r_{2}\right]-\frac{1+r_{3}}{T_{1}},
\end{aligned}
$$

where the spatially averaged visible and dark photon fields are together defined as

$$
\bar{E}_{1}^{\prime}=\bar{E}_{1}+\chi \eta \bar{E}^{\prime}, \quad \bar{E}_{2}^{\prime}=\bar{E}_{2}+\chi \eta \bar{E}^{\prime}
$$

and we assume that $a_{e g}$ is real. Note that in the expression above we assumed that $\bar{E}_{1}$ and $\bar{E}_{2}$ are in phase, which is appropriate for atoms pumped by phase-matched lasers. Due to the smallness of the mixing parameter $\chi$, the dark photon field itself will not drive the evolution of the state population in the system. However, the dark photon can trigger the production of $E_{1}$ and $E_{2}$, which in turn trigger additional photon production. Therefore, while it would be safe to drop the dark photon component in Eqs. (88)-(90), we retain it in numeric computations for the sake of rigor.

Of course, because it is essential to the development of electromagnetic fields in $\mathrm{pH}_{2}$, we must retain the dark photon component in the field equations. Using Eqs. (85)-(87), we obtain

$$
\begin{aligned}
\left(\partial_{t}-\partial_{z}\right) E_{1}= & \frac{i \omega n}{2}\left[\left(\frac{a_{e e}+a_{g g}}{2}+\frac{a_{e e}-a_{g g}}{2} r_{3}\right) E_{1}\right. \\
& \left.+a_{e g}\left(r_{1}-i r_{2}\right)\left(E_{2}^{*}+\chi \eta E^{*}\right)\right], \\
\left(\partial_{t}+\partial_{z}\right) E_{2}= & \frac{i \omega n}{2}\left[\left(\frac{a_{e e}+a_{g g}}{2}+\frac{a_{e e}-a_{g g}}{2} r_{3}\right)\left(E_{2}+\chi \eta E^{\prime}\right)\right. \\
& \left.+a_{e g}\left(r_{1}-i r_{2}\right) E_{1}^{*}\right], \\
\left(\partial_{t}+\partial_{z}\right) E^{\prime}= & \frac{i \omega n}{2}\left[\left(\frac{a_{e e}+a_{g g}}{2}+\frac{a_{e e}-a_{g g}}{2} r_{3}\right)\left(2 \chi^{2} \eta E^{\prime}+\chi E_{2}\right)\right. \\
& \left.\left.+a_{e g}\left(r_{1}-i r_{2}\right) \chi \eta E_{1}^{*}\right)\right] .
\end{aligned}
$$

In the experimental setup described below, after the atoms are pumped into their excited states, the laser fields will be shut off so that $\left|\tilde{E}_{1}\right|=\left|\tilde{E}_{2}\right| \approx 0$. It is clear from Eq. (91) that in this circumstance, a nonzero dark electric field $E^{\prime}$ will be essential to develop the $E_{1}$ field, which will in turn trigger additional two-photon emission.

\section{DETECTING DARK-PHOTON-INDUCED TWO-PHOTON TRANSITIONS}

\section{A. Experimental setup}

Our proposed experimental setup is schematically illustrated in Fig. 5. A continuous laser beam is injected into a resonant cavity, which enhances the laser's probability to oscillate into dark photons. After hitting the wall, photons are stopped and only dark photons are allowed through. The target $\left(\mathrm{pH}_{2}\right.$, for example) is pumped into a coherently excited state, as detailed in Sec. II. As it propagates through the target, the dark photon field triggers atomic deexcitation. The electric fields generated from the first deexcitation subsequently trigger two-photon emission, producing backto-back photons with the same frequency. These photons trigger further deexcitations, detected at both ends of the target vessel.

There are two primary advantages to conducting the experiment in the manner described above. First, the $\mathrm{pH}_{2}$ sample's response to a dark photon field can be precisely determined by passing a very weak laser field through the sample, where low-power lasers can directly test the response to weakly coupled dark photons. Then, in discovery mode, where visible photons are prevented from passing through the wall, the two-photon emission process would presumably only occur if triggered by a dark photon over the $\sim 10$ ns coherence time, because the spontaneous deexcitation process is negligibly slow, as detailed in Sec. IV. D. Photons produced by dark-photon-induced transitions would be emitted back to back and at the frequency $\omega=\omega_{e g} / 2$. Altogether, this provides a powerful 


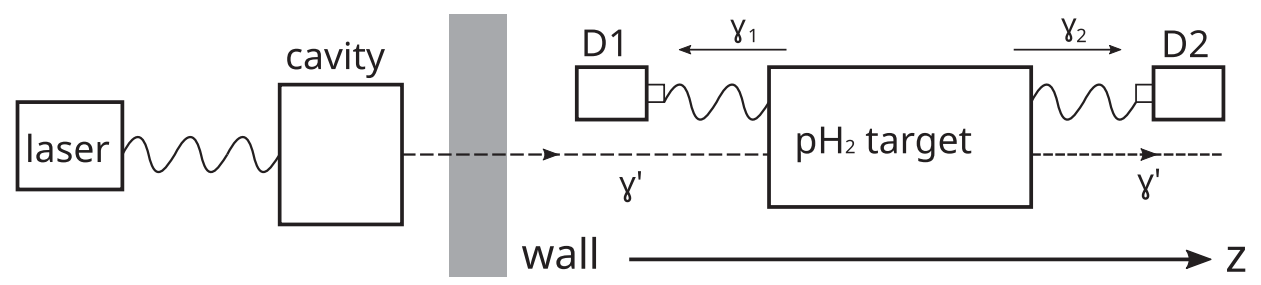

FIG. 5. Schematic view of the proposed experiment. First, the $\mathrm{pH}_{2}$ sample is coherently excited to energy $\omega_{\text {eg }}$ by back-to-back pump lasers (pump lasers not shown). The excited atoms' $E 1$ dipole transitions are parity forbidden, meaning the atoms are metastable over the $\sim 10 \mathrm{~ns}$ integration time of the experiment. On the other hand, the emission of two $\omega_{\text {eg }} / 2$ energy photons in an $E 1 \times E 1$ transition is allowed. As in light-shining-through-wall experiments, a laser is fed into a resonant cavity to increase the dark photon conversion probability. In this case, the laser will operate at energy $\omega_{e g} / 2$, so that after passing through the wall, dark photons act as a trigger field for the emission of back-to-back photons which are then observed by detectors labeled D1 and D2.

background rejection method, since the rate for spontaneous two-photon emission is very small. This can be contrasted with more ambitious experiments utilizing twophoton emission processes $[40,45,49,50]$. In these experiments, the signal (one photon and either two neutrinos or an axion) will need to be distinguished from a sizable twophoton emission background, since both processes are triggered. Therefore, it is plausible that the experiment we have outlined is an intermediate step that could be reached while working towards the proposals laid out in Refs. [40,45,49,50].

\section{B. Dark-photon-induced transition rate}

To begin with, let us quote the estimated rate for the emission of $\gamma_{1}$ and $\gamma_{2}$ in our proposed experiment. First, we note that without both coherent enhancement and exponential amplification of photon fields by the atomic medium (which will be discussed shortly), the $|e\rangle \rightarrow|g\rangle+$ $\gamma+\gamma^{\prime}$ transition rate depicted in Fig. 4(a) is rather slow. To satisfy the coherent amplification condition, we require

$$
(\omega-k) L \lesssim 1
$$

where $L$ is the length of the target, which is the longest dimension of the target volume.

Under these conditions (see Appendix B for a full derivation), the naive rate for dark-photon-induced twostage transitions is

$$
\begin{aligned}
\Gamma_{\gamma^{\prime} \gamma}= & \frac{1}{4 \pi}\left(N_{\text {pass }}+1\right) \chi^{4} \sin ^{2}\left(\frac{m_{A^{\prime}}^{2}}{4 \omega^{\prime}} l\right) \\
& \times P_{L}|\eta|^{2}\left|a_{e g}\right|^{2}\left|\rho_{g e}\right|^{2} n^{2} V^{2} \omega_{1}^{3},
\end{aligned}
$$

where $\omega_{1}$ is the cavity laser frequency, equal to the dark photon frequency $\omega^{\prime}, N_{\text {pass }}$ is the number of cavity reflections, $P_{L}$ is the cavity laser power, $l$ is the cavity length, $A$ is the area of the excited atomic target (limited by the pump lasers' beam width), and $n$ is the target number density. In Table II we give the parameters for the laser cavity and parahydrogen sample in our benchmark setup.

Using this naive estimate results in an unobservably small rate, because it does not account for the development of electromagnetic fields in the atomic medium [cf. the field equations given in Eqs. (91)-(93)]. The predicted rate for our benchmark experimental and model parameters given in Table II, for a dark photon mixing $\chi=10^{-9}$ and mass $m_{A^{\prime}}=10^{-4} \mathrm{eV}$, and for parahydrogen dipole coupling $a_{e g}=0.0275 \times 10^{-24} \mathrm{~cm}^{3}$, is $\Gamma \approx 10^{-5} \mathrm{~s}^{-1}$. This emission rate is unobservably low considering that each experimental run is expected to last about $10 \mathrm{~ns}$.

However, even a small production rate for $E_{1}$ can be exponentially enhanced in coherently prepared atoms. As detailed in Appendix A, the transition rate for producing two photon pairs is exponentially enhanced as the electromagnetic field strength grows,

$$
\Gamma_{\text {sup }}=\frac{1}{16 \pi}\left|a_{e g}\right|^{2}\left|\rho_{g e}\right|^{2} N^{2} V \omega_{1}^{2}\left|E_{1}\right|^{2}\left|E_{2}\right|^{2} .
$$

\begin{tabular}{|c|c|}
\hline Dark Photon Generating Cavity & Superradiant Parahydrogen Target \\
\hline $\begin{array}{l}\text { Cavity Length } l=50 \mathrm{~cm} \\
\text { Cavity Reflections } N_{\text {pass }}=2 \times 10^{4} \\
\text { Cavity Laser Frequency } \omega^{\prime}=0.26 \mathrm{eV} \\
\text { Cavity Laser Power } P_{L}=1 \mathrm{Wm}^{-2} \\
\ldots\end{array}$ & $\begin{array}{c}\text { Sample Length } L=30 \mathrm{~cm} \\
\mathrm{pH}_{2} \text { Density } n=10^{21} \mathrm{~cm}^{-3} \\
\text { Pump Laser Frequency } \omega_{1}=0.26 \mathrm{eV} \\
\text { Pump Laser Power } \approx 10^{9} \mathrm{~W} \mathrm{~mm}-2 \\
\mathrm{pH}_{2} \text { Sample Area } A=1 \mathrm{~cm}^{2}\end{array}$ \\
\hline
\end{tabular}

TABLE II. Parameters for our benchmark experimental setup. For the dark-photon-generating laser cavity, we take parameters matching those of the ALPS experiment [17]. For the $\mathrm{pH}_{2}$ sample, we quote values necessary to obtain maximum coherence, as investigated in Sec. II. 
The dependence on $E_{1}^{2} E_{2}^{2}$ in Eq. (96) shows that the growth of signal fields will be exponential after the dark photon establishes a small $E_{1}$ seed field. A similar amplification has been observed to be as large as $10^{18}$ compared with spontaneous emission [36]. We expect an even larger amplification factor to be achieved in our benchmark experimental setup.

\section{Numerically simulating field development}

When simulating the development of electric fields in coherently prepared atoms, it will be convenient to rescale the spacetime coordinates and the electric fields to be dimensionless. We define

$$
\begin{aligned}
\beta & =\frac{2}{n \omega_{e g} a_{e g}}, \quad \zeta=\frac{z}{\beta}, \quad \tau=\frac{t}{\beta}, \\
\left|e_{1(2)}\right|^{2} & =\frac{\left|E_{1(2)}\right|^{2}}{\omega_{e g} n}, \quad\left|e^{\prime}\right|^{2}=\frac{\left|E^{\prime}\right|^{2}}{\omega_{e g} n},
\end{aligned}
$$

where $\beta$ represents the typical length and time scale for the evolution of the system and $\omega_{e g} n$ is the energy stored in excited atoms. The Bloch equations and field equations can be written in terms of these new variables:

$$
\begin{aligned}
\partial_{\tau} r_{1}=[ & \left.-\frac{a_{g g}-a_{e e}}{2 a_{e g}}\left(\left|e_{1}\right|^{2}+\left|e_{2}\right|^{2}\right)+\beta \delta\right] r_{2} \\
+ & 2 \operatorname{Im}\left(e_{1} e_{2}\right) r_{3}-\frac{r_{1}}{\tau_{2}}, \\
\partial_{\tau} r_{2}= & {\left[\frac{a_{g g}-a_{e e}}{2 a_{e g}}\left(\left|e_{1}\right|^{2}+\left|e_{2}\right|^{2}\right)-\beta \delta\right] r_{1} } \\
& +2 \operatorname{Re}\left(e_{1} e_{2}\right) r_{3}-\frac{r_{2}}{\tau_{2}}, \\
\partial_{\tau} r_{3}=-2[ & \left.\operatorname{Im}\left(e_{1} e_{2}\right) r_{1}+\operatorname{Re}\left(e_{1} e_{2}\right) r_{2}\right]-\frac{1+r_{3}}{\tau_{1}}, \\
\left(\partial_{\tau}-\partial_{\zeta}\right) e_{1}= & \frac{i}{2}\left[\left(\frac{a_{e e}+a_{g g}}{2 a_{e g}}+\frac{a_{e e}-a_{g g}}{2 a_{e g}} r_{3}\right) e_{1}\right. \\
& \left.+\left(r_{1}-i r_{2}\right)\left(e_{2}^{*}+\chi \eta e^{\prime *}\right)\right], \\
\left(\partial_{\tau}+\partial_{\zeta}\right) e_{2}= & \frac{i}{2}\left[\left(\frac{a_{e e}+a_{g g}}{2 a_{e g}}+\frac{a_{e e}-a_{g g}}{2 a_{e g}} r_{3}\right)\left(e_{2}+\chi \eta e^{\prime}\right)\right. \\
& \left.+\left(r_{1}-i r_{2}\right) e_{1}^{*}\right], \\
+ & \left.\left.\left(r_{1}-i r_{2}\right) \chi \eta e_{1}^{*}\right)\right] . \\
\left(\partial_{\tau}+\partial_{\zeta}\right) e^{\prime}= & \frac{i}{2}\left[\left(\frac{a_{e e}+a_{g g}}{2 a_{e g}}+\frac{a_{e e}-a_{g g}}{2 a_{e g}} r_{3}\right)\left(2 \chi^{2} \eta e^{\prime}+\chi e_{2}\right)\right.
\end{aligned}
$$

As mentioned before, the dipole couplings of parahydrogen have been measured to be $a_{g g}=0.90 \times 10^{-24}$, $a_{e e}=0.87 \times 10^{-24}$, and $a_{e g}=0.0275 \times 10^{-24} \mathrm{~cm}^{3}[41]$. For the relaxation and decoherence times, we take $T_{1}=$ $10^{3}$ and $T_{2}=10 \mathrm{~ns}$, respectively; for an extended discussion of coherence in preparations of $\mathrm{pH}_{2}$, see Sec. II. The photon and dark photon energies are $\omega=\omega_{e g} / 2 \approx 0.26 \mathrm{eV}$. Altogether, this gives

$$
\begin{aligned}
\beta & =0.092\left(\frac{10^{21} \mathrm{~cm}^{-3}}{n}\right) \mathrm{ns}=2.8\left(\frac{10^{21} \mathrm{~cm}^{-3}}{n}\right) \mathrm{cm}, \\
\omega_{e g} n & =2.5 \times 10^{10}\left(\frac{n}{10^{21} \mathrm{~cm}^{-3}}\right) \mathrm{W} / \mathrm{mm}^{2} .
\end{aligned}
$$

A typical target vessel is 10 to $100 \mathrm{~cm}$ long. Here we assume a vessel that is $30 \mathrm{~cm}$ long, which is smaller than the expected length scale over which the $\mathrm{pH}_{2}$ is coherent. If we assume that all atoms are initially prepared in the coherent state, then $r_{1}=1$ across the target. We also consider smaller values of $r_{1}=0.1,0.5,0.9$, which correspond to fewer atoms in the coherent state. With the aid of a resonant cavity, the transmission probability for a dark photon to shine through the wall is given by $[64,65]$

$$
p_{\text {trans }}=2\left(N_{\text {pass }}+1\right) \chi^{2} \sin ^{2}\left(\frac{m_{A^{\prime}}^{2}}{4 \omega} l\right)
$$

where $l$ is the size of the cavity and $N_{\text {pass }}$ is the number of reflections the laser undergoes in the dark-photon-generating cavity. We assume that the laser cavity has the parameters given in Table II; these values are in line with what has been attained at the ALPS II experiment [17]. The initial dark photon field power in the target volume is estimated to be

$$
\left|E^{\prime}(t=0)\right|^{2}=P_{\mathrm{L}} p_{\text {trans }},
$$

where for our benchmark setup we assume a laser power $P_{\mathrm{L}}=1 \mathrm{~W} \mathrm{~mm}^{-2}$. As mentioned in Sec. III. B. $1, \eta$ is determined by the relative phase between the polarization of the photon and dark photon fields. Without loss of generality we set it to unity.

In Figs. 6-8 we show the time evolution of the system. In these figures we assume that all of the $\mathrm{pH}_{2}$ atoms are initially prepared in the coherent state, i.e., $r_{1}=1, r_{2}=0$, and $r_{3}=0$, across the target. We also assume a dark photon mass $m_{A^{\prime}}=0.1 \mathrm{meV}$.

As shown in Fig. 6, $r_{1}$ and $r_{3}$ decay exponentially when no laser is present. In this case no initial dark photon field is pumped through the wall and so spontaneous deexcitation dominates the evolution of the system. We note that Fig. 7, which shows no substantial $E_{1}, E_{2}$, or $E^{\prime}$ field developing when $P_{L}=0$, does not include the effect of spontaneous two-photon deexcitations, which are expected to be negligibly small; see Sec. IV. D. 

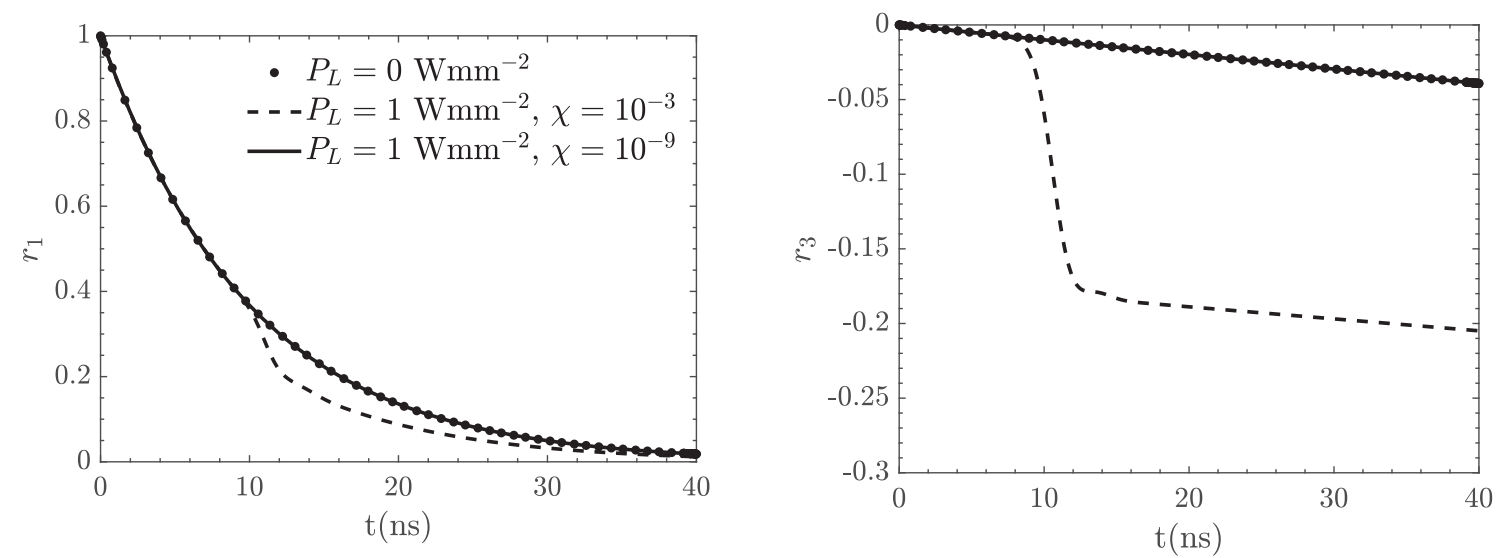

FIG. 6. Time evolution of Bloch vectors at the center of the target. Left panel: $r_{1}$ as a function of time. Right panel: $r_{3}$ as a function of time. Dotted lines show the case where no initial cavity laser is present to create a dark photon $\left(P_{L}=0\right)$. The dashed and solid lines correspond to dark photon mixing parameters $\chi=10^{-3}$ and $10^{-9}$, respectively, for a cavity laser generating dark photons with power $P_{L}=1 \mathrm{~W} \mathrm{~mm} m^{-2}$. A maximally coherent parahydrogen sample, $r_{1}(t=0)=1, r_{2}(t=0)=0, r_{3}(t=0)=0$, and a dark photon mass $m_{A^{\prime}}=0.1 \mathrm{meV}$ are assumed.
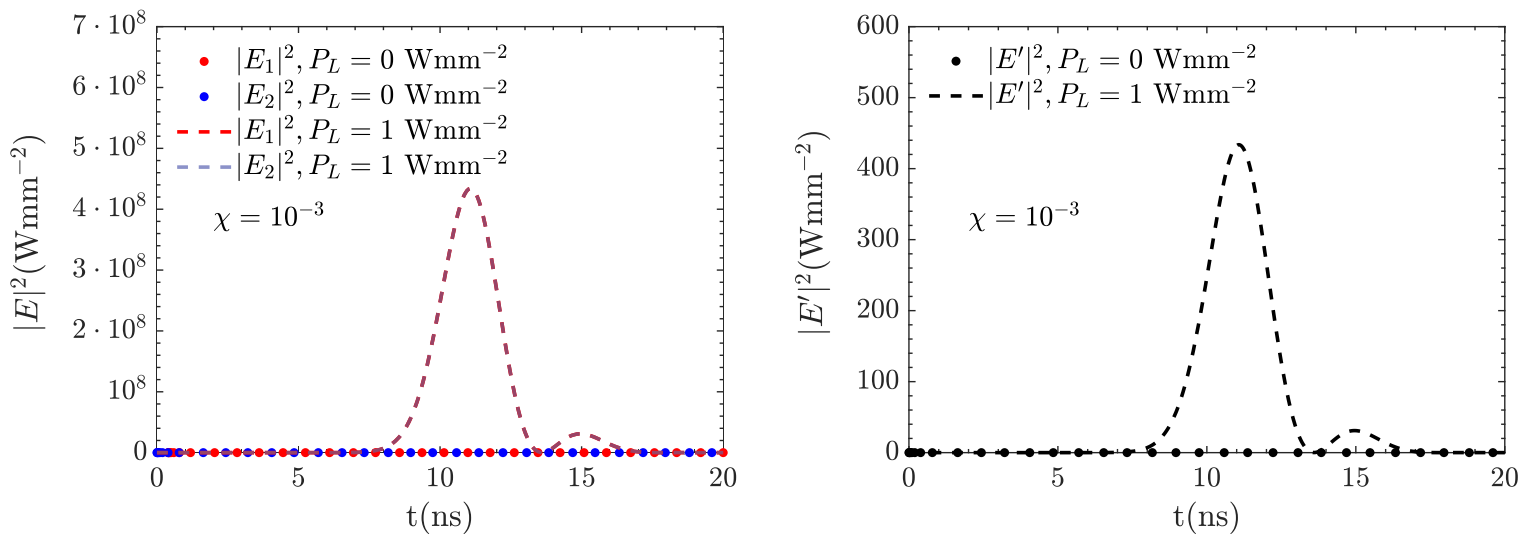

FIG. 7. Time evolution of the (dark) electric fields at the ends of the parahydrogen target. Left panel: The electric fields at each end of the target, $\left|E_{1}\right|^{2}$ and $\left|E_{2}\right|^{2}$, as a function of time. Right panel: The dark photon field $\left|E^{\prime}\right|^{2}$ as a function of time. As in Fig. 6, the cavity laser is turned off $\left(P_{L}=0\right)$ for the dotted lines and a $P_{L}=1 \mathrm{~W} \mathrm{~mm}^{-2}$ cavity laser with dark photon mixing $\chi=10^{-3}$ is assumed for the dashed lines. We also assume the same initial Bloch vectors as in Fig. 6. $\left|E_{1}\right|^{2}$ (red) is taken at the left end of the target with $z=0 \mathrm{~cm}$, while $\left|E_{2}\right|^{2}$ (blue) and $\left|E^{\prime}\right|^{2}$ (black) are taken at the right end of the target with $z=30 \mathrm{~cm}$.
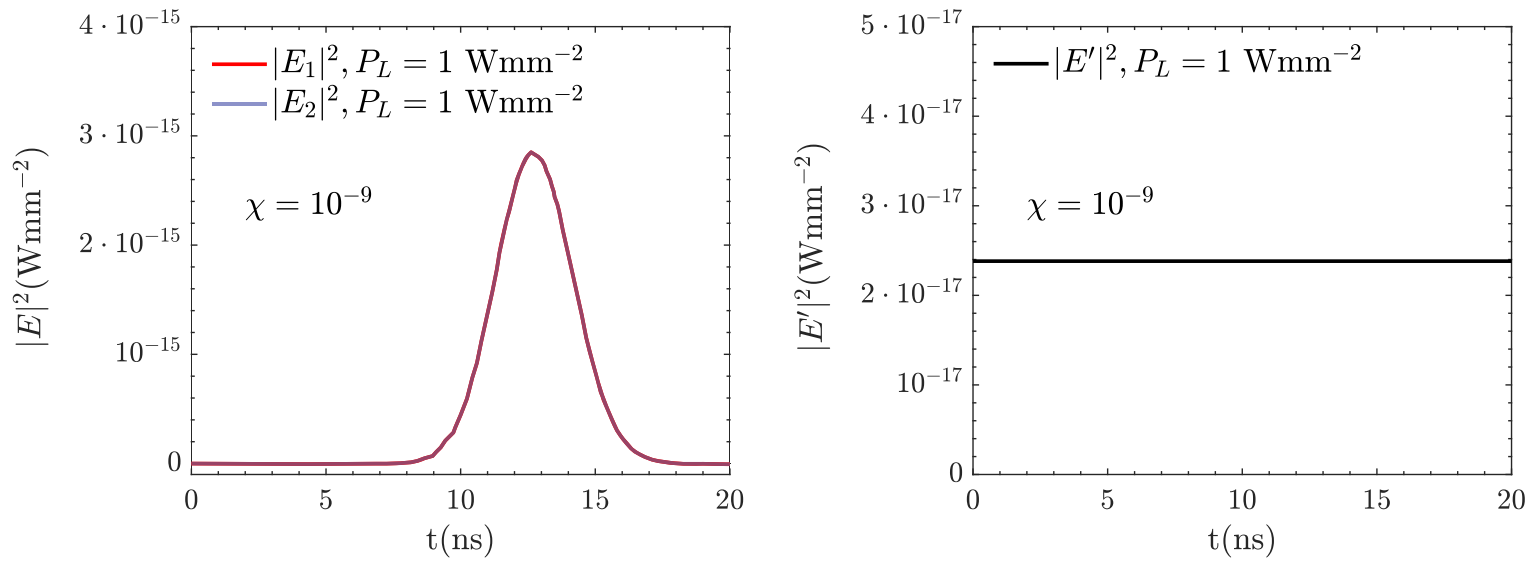

FIG. 8. Time evolution of the (dark) electric fields at the ends of the target. Other features are the same as in Fig. 7, except that a smaller dark photon mixing $\chi=10^{-9}$ is used and $\left|E_{1}\right|^{2},\left|E_{2}\right|^{2}$, and $\left|E^{\prime}\right|^{2}$ are shown with solid lines. 
This scenario changes dramatically in the presence of dark photons produced by a laser. Assuming the laser power $P_{L}=1 \mathrm{~W} \mathrm{~mm}^{-2}$ and the mixing $\chi=10^{-3}$, a sudden drop takes place in $r_{1}$ and $r_{3}$ around $10 \mathrm{~ns}$. This drop corresponds to decay and release of the target's energy through the production of $E_{1}$ and $E_{2}$ as well as a minor enhancement of the dark photon field $E^{\prime}$. The dynamics can be explained as follows. The initial dark photon field induces a deexcitation via $E_{1}$ and $E^{\prime}$ [Fig. 4(a) illustrates this process], and then this $E_{1}$ field triggers additional twophoton deexcitation producing $E_{1}$ and $E_{2}$ symmetrically [see Fig. 4(b)]. The growing $E_{1}$ and $E_{2}$, when large enough, cause abrupt decoherence and deexcitation, which in turn gives rise to additional energy release in the form of $E_{1}$ and $E_{2}$. As can be identified from Eq. (87), $E^{\prime}$ will also be generated by $E_{1}$-induced transitions, at a rate suppressed by $\chi$.

The transitions are less explosive when $\chi=10^{-9}$, as illustrated in Figs. 6 and 8. The deviations of $r_{1}$ and $r_{3}$ from spontaneous decay are barely observed and the peak intensity of $E_{1}$ and $E_{2}$ are relatively low compared to $\chi=10^{-3}$. In this case, the dark photon has induced the generation of an observable but small quantity of $E_{1}$ and $E_{2}$ photons. The dark photon field remains essentially constant since $E^{\prime}$ regenerated from $E_{1}$ is too small to be observed.

\section{Spontaneous two-photon emission background}

We now consider a possible background from spontaneous deexcitation and emission of photons from cold atoms over the runtime of the proposed experiment (around $10 \mathrm{~ns})$. We will find that this background is negligible. Since the transition from the excitation state $|e\rangle$ to the ground state $|g\rangle$ is $E 1$ forbidden, single-photon deexcitation is only viable through higher-order transitions. Note that we are only looking for signal photons with energy around $\omega=\frac{1}{2} \omega_{e g}$, because our signal photons are expected at this frequency. The background from spontaneous two-photon emission has a rate given by (see Appendix A)

$\frac{d \Gamma_{\mathrm{sp}}}{d z}=\frac{\omega_{e g}^{7}}{(2 \pi)^{3}} N\left|a_{e g}\right|^{2} z^{3}(1-z)^{3}=1.27 \times 10^{-14} \mathrm{~s}^{-1}$,

where $z=\omega_{1} / \omega_{\text {eg }}$ is the fraction of the energy for one of the two photons in the transition. We assume an uncertainty $\Delta \nu=100 \mathrm{MHz}$ in the frequency measurement, which translates to $\Delta z=8.0 \times 10^{-7}$. For a sample target with length $L=30 \mathrm{~cm}$ and cross section area $A=1 \mathrm{~cm}^{2}$, the uncertainty in the emission solid angle is $\Delta \Omega / 4 \pi=$ $A / 4 \pi(L / 2)^{2}=3.5 \times 10^{-4}$. These two photons from the spontaneous decay process can be emitted in any direction. Since we only detect photons at the ends of the atomic sample, the fraction of background photons that reach the detector is $2 \Delta \Omega / 4 \pi$. Given the target number density $n=$ $10^{21} \mathrm{~cm}^{-3}$ and complete coherence $\left(\rho_{e g}=0.5\right)$, the total number of $\mathrm{pH}_{2}$ atoms in the excitation state for our benchmark setup is $N=1.5 \times 10^{22}$. Even given a generously long measurement time $\Delta t=40 \mathrm{~ns}$, the spontaneous two-photon emission background is estimated to be

$$
N_{\text {background }}=2 N \frac{d \Gamma_{\mathrm{sp}}}{d z} \Delta z \Delta t \frac{\Delta \Omega}{4 \pi}=4.3 \times 10^{-9} .
$$

We see that over the course of any reasonable number of experimental repetitions, we should not expect a single background event from spontaneous two-photon deexcitation processes.

\section{E. Results and sensitivity}

The signature of the proposed dark photon search is the symmetric emission of photons with frequency $\omega=\omega_{\text {eg }} / 2$ at both ends of the target. The number of signal photons emitted during one experimental trial run (of $\sim 10 \mathrm{~ns}$ ) is

$$
N_{s}^{1}=\frac{A}{\omega} \int_{0}^{t}\left|E_{1}\left(t^{\prime}\right)\right|^{2} d t^{\prime}=\frac{A}{\omega} \int_{0}^{t}\left|E_{2}\left(t^{\prime}\right)\right|^{2} d t^{\prime},
$$

where $A$ is the area of the target and $t$ is the time duration of the experiment. The experiment can be repeated many times to accumulate signal photons. The Bloch equations and field equations derived in Sec. III are highly nonlinear, but we see from Eq. (91) that $E_{1} \propto \chi E^{\prime}$, and therefore the number of photons emitted is

$$
N_{s} \propto P_{L} N_{\text {rep }} \chi^{4}\left(N_{\text {pass }}+1\right) \sin ^{2}\left(\frac{m_{A^{\prime}}^{2}}{4 \omega} l\right),
$$

where $N_{\text {rep }}$ is the number of repetitions of the experiment. To see in what regime this scaling holds, we show in Fig. 9 the number of photons produced as a function of the mixing $\chi$ assuming different laser powers. Note that in the limit that $m_{A^{\prime}}^{2} \ll \frac{4 \omega}{l}$, the expected number of signal photons scales like $N_{s} \propto \chi^{4} m_{A^{\prime}}^{4}$, as is evident in Fig. 9. There is an upper bound on the number of signal photons, which is saturated if all of the excited atoms are deexcited. It is clear from the figure that before saturation $N_{s}$ is proportional to $P_{L}$ and $\chi^{4}$. As $\chi$ becomes large enough, a significant amount of energy stored in the target is released and one gains very little by increasing the mixing or laser power. We also note that $N_{\text {rep }}, N_{\text {pass }}+1$, and $\sin ^{2}\left(m_{A^{\prime}}^{2} l / \omega\right)$ will have the same scaling as $P_{L}$ when determining the number of signal photons emitted.

To estimate the sensitivity of our proposed experiment, we require the emission of at least ten photon pairs after a certain number of excitation/deexcitation repetitions. As a benchmark we take the laser power $P_{L}=1 \mathrm{~W} \mathrm{~mm}^{-2}$, target area $A=1 \mathrm{~cm}^{2}$, target chamber length $L=30 \mathrm{am}$, number of dark-photon-generating cavity reflections $N_{\text {pass }}=2 \times 10^{4}$, cavity size $l=50 \mathrm{~cm}$, and number of experimental 


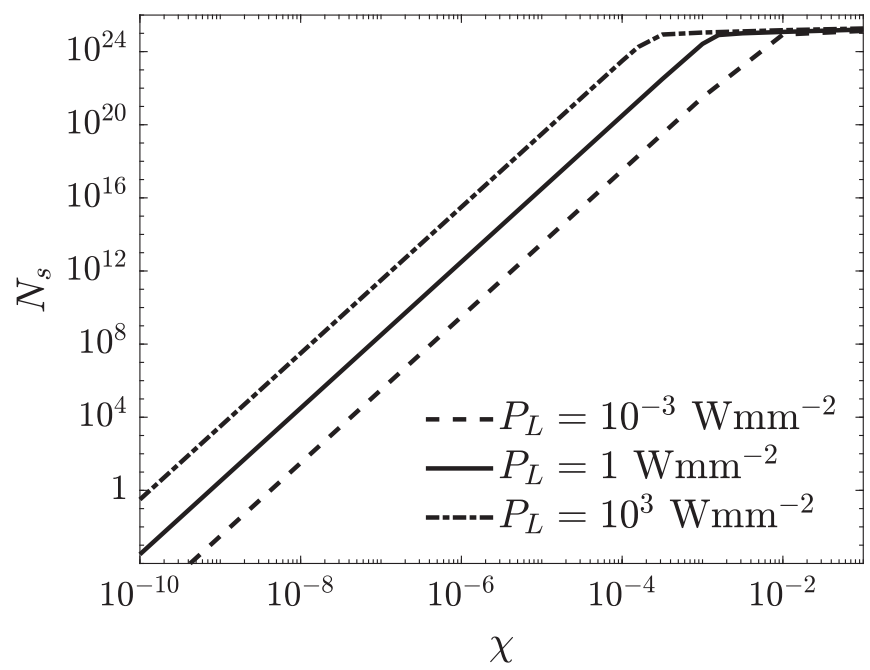

FIG. 9. Number of photons emitted as a function of $\chi$ at the ends of the target volume, for different $P_{L}$, where this is the power of the laser producing dark photons in the reflection cavity. The dashed, solid, and dash-dotted lines correspond to dark photon cavity laser powers $P_{L}=10^{-3}, 1$, and $10^{3} \mathrm{~W} \mathrm{~mm}^{-2}$, respectively. Otherwise, we take the benchmark parameters shown in Table II: the number of cavity reflections $N_{\text {pass }}=2 \times 10^{4}$, the cavity length $l=50 \mathrm{~cm}$, the dark photon mass $m_{A^{\prime}}=0.1 \mathrm{meV}$, the laser frequency $\omega=0.26 \mathrm{eV}$, the area of the parahydrogen target $A=1 \mathrm{~cm}^{2}$, and the number of experimental repetitions (each around $10 \mathrm{~ns}$ ) is $N_{\text {rep }}=10^{3}$. The initial Bloch vectors are the same as in Fig. 6.

repetitions $N_{\text {rep }}=10^{3}$. In the regime where a fraction of the $\mathrm{pH}_{2}$ deexcites, the number of emitted photons can be estimated as

$$
\begin{aligned}
N_{s}= & 5 \times 10^{3} \frac{P_{L}}{1 \mathrm{~W} \mathrm{~mm}^{-2}}\left(\frac{\chi}{10^{-9}}\right)^{4} \frac{N_{\text {pass }}+1}{2 \times 10^{4}+1} \\
& \times \sin ^{2}\left(1.27 \frac{m_{A^{\prime}}^{2}}{\mathrm{meV}^{2}} \frac{\mathrm{eV}}{\omega} \frac{l}{m}\right),
\end{aligned}
$$

where this expression has been normalized assuming $n=10^{21} \mathrm{~cm}^{-3}$.

We show the sensitivity of our proposed experiment in Fig. 10. Also shown in the figure are the light-shiningthrough-wall experiments, and cosmological and astrophysical bounds reviewed in Ref. [66]. The coherent amplification condition we have assumed throughout given by Eq. (94) requires that our dark photon mass not be too large. This restricts $m_{A^{\prime}} \lesssim 0.6 \mathrm{meV}$. As a consequence, we have truncated the mass sensitivity at $1 \mathrm{meV}$. As seen from the figure, over the mass range $10^{-5}-10^{-3} \mathrm{eV}$ our proposed experiment appears rather sensitive to dark photon kinetic mixing. Note that so far we have assumed that the detuning $\delta=0$. A nonzero detuning has a mild effect on the expected sensitivity. We refer the reader to Appendix C for a quantitative discussion.

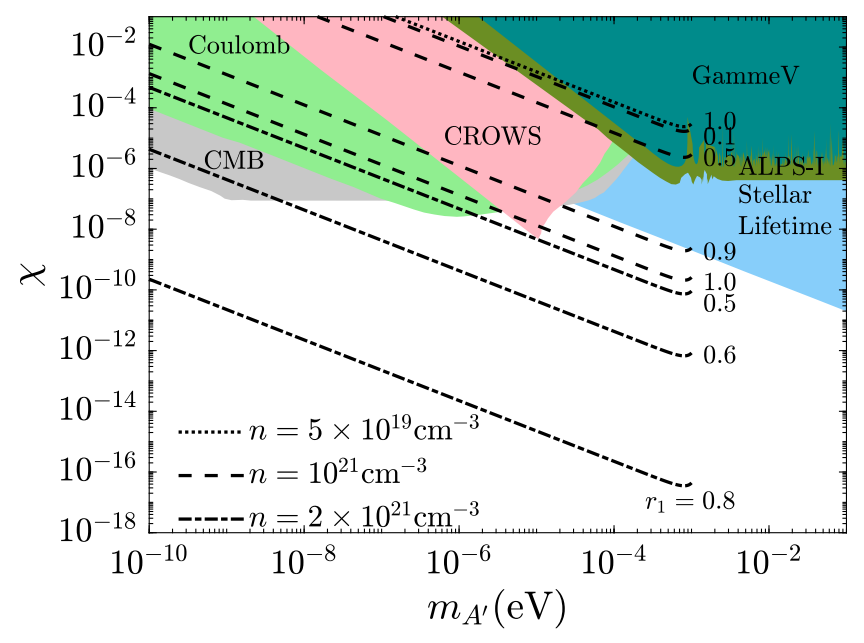

FIG. 10. The sensitivity of our proposed experiment assuming the benchmark parameters given in Table II, except for the $\mathrm{pH}_{2}$ target number density $n$ and Bloch vector $r_{1}(t=0)$ as indicated. We also assume $r_{2}(t=0)=r_{3}(t=0)=0$. The experiment is repeated $N_{\text {rep }}=10^{3}$ times by coherently exciting the $\mathrm{pH}_{2}$ sample, where each coherent excitation lasts $\sim 10 \mathrm{~ns}$. The constraints from other dark photon experiments, astrophysics, and cosmology are shown for comparison: Coulomb [67,68], CMB [69,70], CROWS [71], GammeV [72], ALPS [16,17], and stellar constraints [10,11,15,17]; see Ref. [66] for a summary of these bounds. The black lines show the sensitivity of our proposed experiment for the $\mathrm{pH}_{2}$ number densities indicated and coherence factors $\left(r_{1}\right)$ as indicated to the right of each sensitivity. Section II and particularly Table I provide a discussion of the coherence that has been achieved in ongoing $\mathrm{pH}_{2}$ experiments [37]: the maximum coherence factor obtained so far is $r_{1} \sim 0.06$. The experimental sensitivities shown are obtained by numerically solving Eqs. (88)-(93), which determine the nonlinear development of coherence induced by counter-propagating lasers in $\mathrm{pH}_{2}$. As has been noted in previous literature, the $\mathrm{pH}_{2}$ transition rate and resulting experimental sensitivity exhibit dramatic nonlinear sensitivity to the $\mathrm{pH}_{2}$ coherence [36-40,43]. This accounts for the dramatic increase in sensitivity between the bottom three sensitivity curves for coherence values $r_{1}=0.5-0.8$. The sensitivity curves have been truncated at a dark photon mass $m_{A^{\prime}}=\mathrm{meV}$, beyond which the coherent amplification condition (94) is no longer valid.

Looking at Fig. 10, it is apparent that the number of signal photons depends on the number density of the target $n$ and the coherence factor $r_{1}$ in a nonlinear and nontrivial manner. To examine this behavior, we can combine Eqs. (91)-(93) and for the moment neglect the propagation terms, $r_{2}$, and the position and time dependence of $r_{1}$ to obtain

$$
\left(\partial_{t}^{2}-\partial_{z}^{2}\right) E_{1}-n^{2} \Omega_{r}^{2} E_{1}=0
$$

where we define $\Omega_{r}^{2} \equiv \omega^{2}\left|a_{e g} r_{1}\right|^{2}\left(1+\chi^{2}|\eta|^{2}\right) / 4$. The temporal part of $E_{1}$ can be solved from Eq. (112), which indicates that $E_{1} \propto e^{n \Omega_{r} t}$. This gives the total photon yield 


$$
N_{s} \propto \int\left|E_{1}\right|^{2} d t \sim \frac{1}{n \Omega} e^{2 n \Omega_{r} \Delta t},
$$

where $\Delta t$ is denotes the time duration of the experiment. The $\chi^{2}|\eta|^{2}$ term in $\Omega_{r}$ shows the dependence on the dark photon mixing parameter and relative polarization, since it is the dark photon that triggers the collective deexcitation of the target molecules. We should also keep in mind that coherence $\left(r_{1}\right)$ dies off quickly after $\Delta t \sim T_{2} \sim 10 \mathrm{~ns}$, which causes the drop in field intensity at $t \gtrsim T_{2}$ depicted in Figs. 7 and 8. Therefore, Eq. (113) is in no way close to an exact solution, but it does show that the signal intensity is enhanced by a factor of $\sim e^{n \Omega_{r} t}$ even for a moderate increase in the number density $n$, coherence $r_{1}$, and coherence time $T_{2}$. A related discussion can be found in Ref. [73]. This exponential evolution behavior can also be understood qualitatively. As mentioned in Sec. IV C, the initial dark photon field triggers the emission of $E_{1}$ and $E_{2}$, which will subsequently trigger more two-photon transitions. The number of photons to be triggered is proportional to the number density of the target, which appears in the exponent of the cascade deexcitation rate.

\section{CONCLUSIONS}

We have studied a new method to detect dark photon fields using resonant two-photon deexcitation of coherently excited atoms. Our proposed experiment combines dark photon production techniques demonstrated by light-shiningthrough-wall experiments with a new detection method: dark photons triggering two-photon transitions in a gas of parahydrogen coherently excited into its first vibrational state. The potential coupling sensitivity to dark photons we project in our benchmark setup is orders of magnitude beyond present limits for $\mu \mathrm{eV}$-meV mass dark photon fields.

A major technical hurdle to realizing this proposal will be the preparation of suitably coherent samples of cold parahydrogen using counter-propagating laser beams. As we examined in Sec. II, the coherence times and $\mathrm{pH}_{2}$ densities necessary have already been achieved in laboratory conditions. It remains to suitably increase the fraction of coherently excited $\mathrm{pH}_{2}$ by using more powerful lasers and colder parahydrogen, as explored in Sec. II. However, even if complete parahydrogen sample coherence is not attained, it would still be possible to realize this proposal by increasing the density of parahydrogen, as explored in Sec. IV. Indeed, although we have not shown it in Fig. 10, the setup we proposed with an increased $\mathrm{pH}_{2}$ number density $\left(2 \times 10^{21}\right)$, assuming completely coherent atoms $\left(r_{1}=1\right)$, can probe kinetic mixings $\chi \ll 10^{-15}$. It may also be possible to realize a proposal similar to the one laid out here using two-photon nuclear transitions and free electron lasers. This might permit detecting dark photons at masses greater than an $\mathrm{eV}$.

Our setup relies on the nonlinear development of electromagnetic fields in coherent atoms, and so our sensitivity estimates have relied on numerical simulations of dark photon and photon cascades in $\mathrm{pH}_{2}$. However, as explained in Sec. IV, the proposed experiment will allow for dark photon detection to be directly calibrated using a low-power trigger laser as an equivalent stand-in for the dark photon field itself. For this reason, although we have focused on the detection of dark photons in this paper, very similar methods could be used to detect axions and other light, electromagnetically coupled particles. We leave this and other uses of multistage atomic transitions to future work.

\section{ACKNOWLEDGMENTS}

We especially thank James Fraser for guidance on nonlinear optics and laser power, along with Junwu Huang, Sam McDermott, Alex Wright, and Aaron Vincent for useful discussions. Research at Perimeter Institute is supported by the Government of Canada through Industry Canada and by the Province of Ontario through the Ministry of Economic Development \& Innovation. A. B., J. B., and N. S. acknowledge the support of the Natural Sciences and Engineering Research Council of Canada.

\section{APPENDIX A: COHERENCE AND NONLINEARITY IN TWO-PHOTON EMISSION}

Let us estimate the transition rate of the two-photon emission process, as illustrated in Fig. 4(b). The transition matrix for the $|e\rangle \rightarrow|g\rangle$ transition is

$$
\begin{aligned}
\langle g|i T| e\rangle \simeq & \mathcal{T} \frac{(-i)^{2}}{2} \int_{-\infty}^{+\infty} d t \int_{-\infty}^{+\infty} d t^{\prime}\left\langle g\left|-\mathbf{d} \cdot \tilde{E}_{2}(t)\right| j\right\rangle\langle j| \\
& -\mathbf{d} \cdot \tilde{E}_{1}\left(t^{\prime}\right)|e\rangle \\
= & (-i)^{2} \int_{-\infty}^{+\infty} d t \int_{-\infty}^{t} d t^{\prime}\left\langle g\left|-\mathbf{d} \cdot \tilde{E}_{2}(t)\right| j\right\rangle\langle j| \\
& -\mathbf{d} \cdot \tilde{E}_{1}\left(t^{\prime}\right)|e\rangle
\end{aligned}
$$

where $\mathcal{T}$ is the time-ordering operator and we write the electric fields as

$\tilde{E}_{m}=\frac{1}{2} E_{m} \epsilon e^{-i \omega_{m} t+i \mathbf{k} \cdot \mathbf{r}}+\frac{1}{2} E_{m}^{*} \epsilon^{*} e^{i \omega_{m} t-i \mathbf{k} \cdot \mathbf{r}}, \quad m=1,2$,

where $\omega_{m}$ and $\mathbf{k}_{m}$ are the energy and momentum of the emitted photons. Integrating over $t^{\prime}$ yields

$$
\begin{aligned}
\langle g|i T| e\rangle \simeq & i \frac{d_{j e} d_{g j}}{\omega_{1}-\omega_{e j}} \frac{E_{1} E_{2}}{4} e^{-i\left(\mathbf{k}_{1}+\mathbf{k}_{2}-\mathbf{k}_{e j}^{a}\right) \cdot\left(\mathbf{r}-\mathbf{r}_{a}\right)} \\
& \times \int_{-\infty}^{+\infty} d t e^{i\left(\omega_{1}+\omega_{2}-\omega_{e g}\right) t}
\end{aligned}
$$

where as before we have defined $\omega_{i k}=\omega_{i}-\omega_{k}$ and $d_{i k}=\left\langle i\left|-\mathbf{d} \cdot \epsilon^{(*)}\right| k\right\rangle . \mathbf{k}_{e j}^{a}$ is the change in the momentum of a specific $\mathrm{pH}_{2}$ after the transition and $r_{a}$ is the spatial 
position of the $\mathrm{pH}_{2}$. We can perform the second time integral and obtain

$$
\langle g|i T| e\rangle=i 2 \pi \delta\left(\omega_{e g}-\omega_{1}-\omega_{2}\right) \mathcal{M}_{a}
$$

with

$$
\begin{aligned}
\mathcal{M}_{a} & =\frac{d_{g j} d_{j e}}{\omega_{j e}+\omega_{1}} \frac{E_{1} E_{2}}{4} e^{-i\left(\mathbf{k}_{1}+\mathbf{k}_{2}-\mathbf{k}_{e g}^{a}\right) \cdot\left(\mathbf{r}-\mathbf{r}_{a}\right)} \\
& =\frac{a_{e g}}{4} E_{1} E_{2} e^{-i\left(\mathbf{k}_{1}+\mathbf{k}_{2}-\mathbf{k}_{e g}^{a}\right)\left(\mathbf{r}-\mathbf{r}_{a}\right)} .
\end{aligned}
$$

First we consider the case that the $\mathrm{pH}_{2}$ is not emitting coherently, which we will call spontaneous two-photon deexcitation. In the case of spontaneous two-photon deexcitation, each $\mathrm{pH}_{2}$ emits two photons with frequencies that are not necessarily $\sim \omega_{e g} / 2$, in contrast with two-photon emission induced by a trigger laser (where the trigger laser frequency used in earlier sections of this paper matched the pump laser frequencies, all of these being $\omega_{e g} / 2$ ). In the spontaneous emission case we sum up the contribution from all $\mathrm{pH}_{2}$ molecules, which gives the emission rate

$$
\begin{aligned}
\Gamma_{\mathrm{sp}}= & \int \frac{d^{3} k_{1}}{(2 \pi)^{3}} \frac{d^{3} k_{2}}{(2 \pi)^{3}} \\
& \times\left|\int d^{3} r \sum_{a=1}^{N} \frac{a_{e g}}{4} \sqrt{\frac{4 \omega_{1} \omega_{2}}{V^{2}}} e^{-i\left(\mathbf{k}_{1}+\mathbf{k}_{2}-\mathbf{k}_{e g}^{a}\right)\left(\mathbf{r}-\mathbf{r}_{a}\right)}\right| \\
& \times 2 \pi \delta\left(\omega_{e g}-\omega_{1}-\omega_{2}\right),
\end{aligned}
$$

where we have explicitly replaced $E_{m}$ by $\sqrt{2 \omega_{m} / V}$ for $m=1,2$, and $N$ is the number of spontaneous emitters. Since the exponential phase is random for each molecule, the product of the phases from different molecules will sum up to zero in the expansion of the square in Eq. (A6). This gives

$$
\Gamma_{\mathrm{sp}}=\int \frac{d^{3} k_{1}}{(2 \pi)^{3}} \frac{d^{3} k_{2}}{(2 \pi)^{3}} N\left|a_{e g}\right|^{2} \frac{\omega_{1} \omega_{2}}{4} 2 \pi \delta\left(\omega_{e g}-\omega_{1}-\omega_{2}\right) .
$$

Carrying out the integral, we find

$$
\frac{d \Gamma_{\mathrm{sp}}}{d \omega_{1}}=\frac{1}{(2 \pi)^{3}} N\left|a_{e g}\right|^{2} \omega_{1}^{3} \omega_{2}^{3} .
$$

If we define $z \equiv \omega_{1} / \omega_{e g}$, Eq. (A8) can be written as

$$
\frac{d \Gamma_{\mathrm{sp}}}{d z}=\frac{\omega_{e g}^{7}}{(2 \pi)^{3}} N\left|a_{e g}\right|^{2} z^{3}(1-z)^{3} .
$$

We use this equation to estimate the two-photon spontaneous emission background in Sec. IV. D.
Next we will estimate the rate for two-photon emission for $\mathrm{pH}_{2}$ pumped and triggered in a manner that allows for macro superradiance. In the presence of appropriately applied background fields, $\mathrm{pH}_{2}$ molecules will tend to emit photons collectively with the same momenta. If the phase $k_{e g}^{a}$ is random for every molecule, the product of the phases would still cancel (as we have derived before) and the rate would be proportional to $N$; however, if the molecules are pumped into the excitation state coherently (by counterpropagating lasers, in the setup we have considered), we can drop the superscript $a$ in $k_{e g}^{a}$ and turn the sum into a spatial integral, i.e.,

$$
\begin{aligned}
\Gamma_{\text {sup }}= & \int \frac{d^{3} k_{1}}{(2 \pi)^{3}} \frac{d^{3} k_{2}}{(2 \pi)^{3}} \\
& \times\left|\int d^{3} r \int d^{3} r_{a} \frac{a_{e g}}{4} \rho_{g e} n E_{1} E_{2} e^{-i\left(\mathbf{k}_{1}+\mathbf{k}_{2}-\mathbf{k}_{e g}\right)\left(\mathbf{r}-\mathbf{r}_{a}\right)}\right|^{2} \\
& \times 2 \pi \delta\left(\omega_{e g}-\omega_{1}-\omega_{2}\right),
\end{aligned}
$$

where $n$ is the number density of the target and $\rho_{g e}$ is the fraction of molecules in the coherent state. In the special case we use two counter-propagating lasers with the same frequency to pump the molecules, $\mathbf{k}_{e g} \approx 0$, although of course this can be spoiled by the lasers' linewidth and other experimental factors, as discussed in Sec. II. For a dense and large enough target, the spatial integral in $r_{a}$ turns into a delta function, which gives

$$
\begin{aligned}
\Gamma_{\text {sup }}= & \int \frac{d^{3} k_{1}}{(2 \pi)^{3}} \frac{d^{3} k_{2}}{(2 \pi)^{3}} \\
& \times\left|\frac{a_{e g}}{4} \rho_{g e} N E_{1} E_{2}(2 \pi)^{3} \delta^{3}\left(\mathbf{k}_{1}+\mathbf{k}_{2}-\mathbf{k}_{e g}\right)\right|^{2} \\
& \times 2 \pi \delta\left(\omega_{e g}-\omega_{1}-\omega_{2}\right),
\end{aligned}
$$

where $N$ is the total number of $\mathrm{pH}_{2}$ molecules in the target. In the case where $\mathbf{k}_{e g} \approx 0$, the delta function forces $\mathbf{k}_{1}+\mathbf{k}_{2}=0$, meaning that the two photons emitted superradiantly have to be back to back and have equal frequency. Since the delta function is squared we replace one by the target volume $V$. Evaluating the integrals yields

$$
\Gamma_{\text {sup }}=\frac{1}{16 \pi}\left|a_{e g}\right|^{2}\left|\rho_{g e}\right|^{2} N^{2} V \omega_{1}^{2}\left|E_{1}\right|^{2}\left|E_{2}\right|^{2} .
$$

Equation (A12) shows that the transition rate in two-photon superradiance is proportional to $N^{2}$ if coherence conditions are met. This can be compared with (out-of-phase) spontaneous two-photon emission described in Eq. (A9), where the rate is instead proportional to $N$. We also see that the rate grows nonlinearly with $E_{1}$ and $E_{2}$, the strength of the background fields. At the onset of superradiance, the emission rate is determined by the power of the trigger laser fields. As the photons from the deexcitation increase the 
strength of the electric fields, the deexcitation rate becomes larger and larger. This exponential growth is clearly seen in Fig. 7.

\section{APPENDIX B: ESTIMATE OF DARK-PHOTON- TRIGGERED TWO-STAGE TRANSITIONS}

Let us now move on to estimate the emission rates of $\gamma_{1}$ and $\gamma_{2}$ in our proposed experiment, as depicted in Fig. 5. Consider the process illustrated in Fig. 4(a). First, the transition matrix for the deexcitation from $|e\rangle$ to $|g\rangle$ via the emission of a dark photon and a photon in the dark photon background is given by

$$
\begin{aligned}
\langle g|i T| e\rangle \simeq & \mathcal{T} \frac{(-i)^{2}}{2} \int_{-\infty}^{+\infty} d t \int_{-\infty}^{+\infty} d t^{\prime}\left\langle g\left|-\mathbf{d} \cdot \tilde{E}^{\prime}(t)\right| j\right\rangle\langle j| \\
& -\mathbf{d} \cdot \tilde{E}_{1}\left(t^{\prime}\right)|e\rangle \\
= & (-i)^{2} \int_{-\infty}^{+\infty} d t \int_{-\infty}^{t} d t^{\prime}\left\langle g\left|-\mathbf{d} \cdot \tilde{E}^{\prime}(t)\right| j\right\rangle\langle j| \\
& -\mathbf{d} \cdot \tilde{E}_{1}\left(t^{\prime}\right)|e\rangle,
\end{aligned}
$$

where $\tilde{E}^{\prime}$ and $E_{1}$ are given in a similar form as in Eq. (A2). With the same algebra as in Appendix A, we obtain

$$
\langle g|i T| e\rangle=i 2 \pi \delta\left(\omega_{e g}-\omega_{1}-\omega^{\prime}\right) \mathcal{M}_{a},
$$

with

$$
\mathcal{M}_{a}=\frac{d_{g j}^{\prime} d_{j e}}{\omega_{j e}+\omega_{1}} \frac{E^{\prime}}{2} \sqrt{\frac{\omega_{1}}{2 V}} e^{-i\left(\mathbf{k}_{1}+\mathbf{k}^{\prime}-\mathbf{k}_{e g}\right) \cdot\left(\mathbf{r}-\mathbf{r}_{a}\right)} .
$$

Note that we have replaced $E_{1}$ by $\sqrt{2 \omega_{1} / V}$ for one photon state. After introducing $a_{e g}$ and $\eta$ as defined in Eqs. (22) and (69), we find

$$
\mathcal{M}_{a}=a_{e g} \eta \frac{E^{\prime}}{2} \sqrt{\frac{\omega_{1}}{2 V}} e^{-i\left(\mathbf{k}_{1}+\mathbf{k}^{\prime}-\mathbf{k}_{e g}\right) \cdot \mathbf{r}_{a}} .
$$

Summing up all coherent atoms, the transition rate is now

$$
\begin{aligned}
\Gamma_{\gamma^{\prime} \gamma}= & \int \frac{d^{3} k_{1}}{(2 \pi)^{3}} \frac{d^{3} k^{\prime}}{(2 \pi)^{3}} \\
& \times\left|\int d^{3} r \int d^{3} r_{a} a_{e g} \eta \rho_{g e} n \frac{E^{\prime}}{2} \sqrt{\frac{\omega_{1}}{2 V}} e^{-i\left(\mathbf{k}_{1}+\mathbf{k}^{\prime}-\mathbf{k}_{e g}\right) \cdot\left(\mathbf{r}-\mathbf{r}_{a}\right)}\right|^{2} \\
& \times 2 \pi \delta\left(\omega_{e g}-\omega_{1}-\omega^{\prime}\right) .
\end{aligned}
$$

After some algebra, we arrive at the transition rate

$$
\Gamma_{\gamma^{\prime} \gamma}=\frac{1}{8 \pi}|\eta|^{2}\left|a_{e g}\right|^{2}\left|\rho_{g e}\right|^{2} N^{2} \omega_{1}^{3}\left|E^{\prime}\right|^{2} .
$$

With the dark photon field power given in Eq. (106), we obtain

$$
\begin{aligned}
\Gamma_{\gamma^{\prime} \gamma}= & \frac{1}{4 \pi}\left(N_{\text {pass }}+1\right) \chi^{4} \sin ^{2}\left(\frac{m_{A^{\prime}}^{2}}{4 \omega^{\prime}} l\right) \\
& \times P_{L}|\eta|^{2}\left|a_{e g}\right|^{2}\left|\rho_{g e}\right|^{2} n^{2} V^{2} \omega_{1}^{3} .
\end{aligned}
$$

For $\quad \chi=10^{-9}, \quad m_{A^{\prime}}=10^{-4} \mathrm{eV}, \quad \omega^{\prime}=\omega_{1}=0.26 \mathrm{eV}$, $N_{\text {pass }}=2 \times 10^{4}, l=0.5 \mathrm{~m}, \eta=1, \mathrm{pH}_{2}$ number density $n=10^{21} \mathrm{~cm}^{-3}$, target area $A=1 \mathrm{~cm}^{2}$, length $L=30 \mathrm{~cm}$, laser power $P_{L}=1 \mathrm{~W} / \mathrm{mm}^{2}$, and $a_{e g}=0.0275 \times 10^{-24} \mathrm{~cm}^{3}$, we obtain

$$
\Gamma_{\gamma^{\prime} \gamma}=1.2 \times 10^{-5} \mathrm{~s}^{-1}
$$

This emission rate is relatively low considering the experimental trial time of about $10 \mathrm{~ns}$, which is determined by the decoherence time. Signal photon production, on the other hand, is enhanced when the dark photon triggers twophoton superradiant transitions. This is discussed in Sec. IV. B.

\section{APPENDIX C: LASER DETUNING}

In this Appendix we study how the development of signal photons is altered if the counter-propagating lasers used to excite the cold parahydrogen sample are substantially detuned. This amounts to varying the detuning parameter $\delta$. The primary effect of $\delta$ is to induce the oscillations in the coherence factor $\rho_{g e}$ (and hence the Bloch vectors $r_{1}$ and $r_{2}$ ), as indicated by Eq. (71). We show the effect of $\delta$ in Fig. 11. As $\delta$ increases, the coherence of $\mathrm{pH}_{2}$ is suppressed, and the output photon flux when $\mathrm{pH}_{2}$ deexcites is correspondingly suppressed. However, as shown, there is no suppression for $\delta<100 \mathrm{MHz}$. We note that detuning $\delta<100 \mathrm{MHz}$ has already been achieved in existing experiments [37]. After increasing the detuning to a value as large as $\delta=10^{4} \mathrm{MHz}$ there is a notable decrease in the expected experimental sensitivity, since the resulting $\mathrm{pH}_{2}$ sample will be less coherent. We note that, as before, the experimental sensitivity and $\mathrm{pH}_{2}$ coherence are obtained by numerically solving the differential equations given in Eqs. (88)-(93), which determines the nonlinear development of coherence in $\mathrm{pH}_{2}$. 

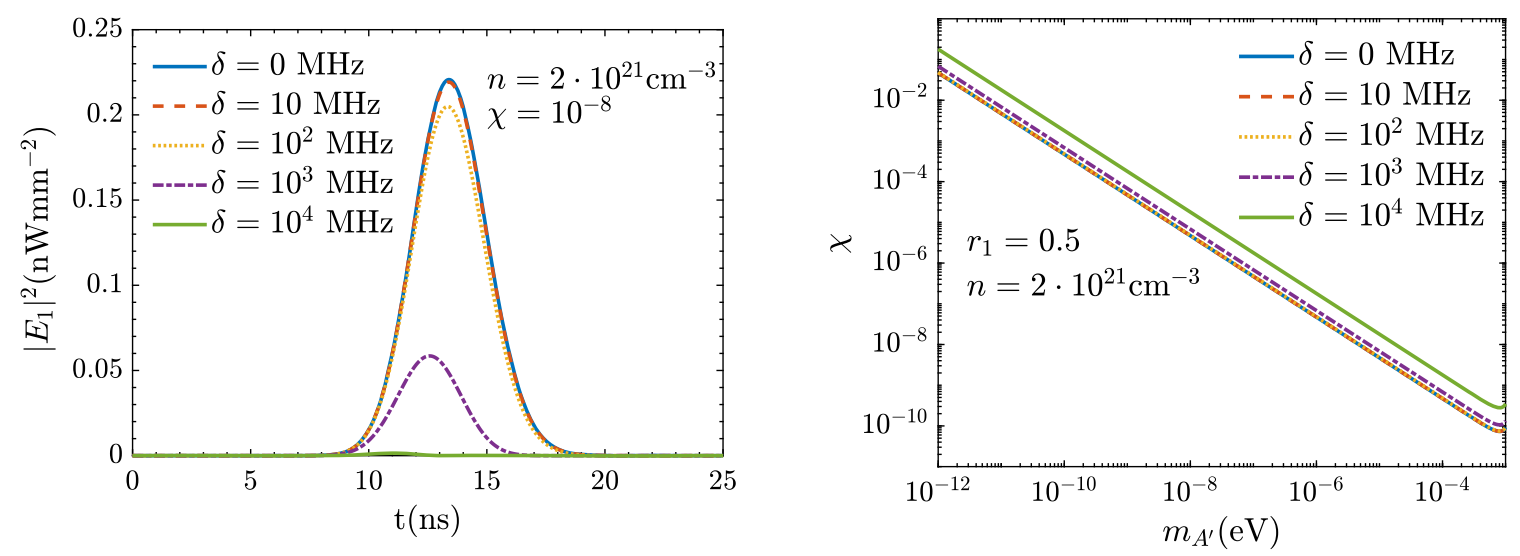

FIG. 11. Effect of varying the detuning parameter. Left panel: Time evolution of the electric field at the end of the $\mathrm{pH}_{2}$ target for $\delta=0$ (solid blue), $\delta=10 \mathrm{MHz}$ (dashed red), $\delta=100 \mathrm{MHz}$ (dotted yellow), $\delta=1000 \mathrm{MHz}$ (dash-dotted purple), and $\delta=10000 \mathrm{MHz}$ (solid green). We assume the benchmark setup given in Table II, except for the $\mathrm{pH}_{2}$ number density $n=2 \times 10^{21} \mathrm{~cm}^{-3}$. We use initial coherence values $r_{1}(t=0)=0.5, r_{2}(t=0)=r_{3}(t=0)=0$, dark photon mixing parameter $\chi=10^{-8}$, and dark photon mass $m_{A^{\prime}}=0.1 \mathrm{meV}$. Note that the electric field power is given in units of $10^{-9} \mathrm{~W} \mathrm{~mm}^{-2}$. Right panel: Projected sensitivity of our proposed experiment for different detunings. The experimental setup is the same as for the left panel.

[1] B. Holdom, Two U(1)'s and Epsilon charge shifts, Phys. Lett. 166B, 196 (1986).

[2] F. del Aguila, G. D. Coughlan, and M. Quiros, Gauge coupling renormalization with several U(1) factors, Nucl. Phys. B307, 633 (1988).

[3] F. del Aguila, M. Masip, and M. Perez-Victoria, Physical parameters and renormalization of U(1)-a x U(1)-b models, Nucl. Phys. B456, 531 (1995).

[4] K. R. Dienes, C. F. Kolda, and J. March-Russell, Kinetic mixing and the supersymmetric gauge hierarchy, Nucl. Phys. B492, 104 (1997).

[5] S. A. Abel, M. D. Goodsell, J. Jaeckel, V. V. Khoze, and A. Ringwald, Kinetic mixing of the photon with hidden U(1)s in string phenomenology, J. High Energy Phys. 07 (2008) 124.

[6] M. Goodsell, J. Jaeckel, J. Redondo, and A. Ringwald, Naturally light hidden photons in LARGE volume string compactifications, J. High Energy Phys. 11 (2009) 027.

[7] M. Pospelov, Secluded U(1) below the weak scale, Phys. Rev. D 80, 095002 (2009).

[8] L. Ackerman, M. R. Buckley, S. M. Carroll, and M. Kamionkowski, Dark matter and dark radiation, Phys. Rev. D 79, 023519 (2009).

[9] N. Arkani-Hamed, D. P. Finkbeiner, T. R. Slatyer, and N. Weiner, A theory of dark matter, Phys. Rev. D 79, 015014 (2009).

[10] H. An, M. Pospelov, and J. Pradler, New stellar constraints on dark photons, Phys. Lett. B 725, 190 (2013).

[11] H. An, M. Pospelov, and J. Pradler, Dark Matter Detectors as Dark Photon Helioscopes, Phys. Rev. Lett. 111, 041302 (2013).

[12] J. Redondo and G. Raffelt, Solar constraints on hidden photons re-visited, J. Cosmol. Astropart. Phys. 08 (2013) 034.
[13] N. Vinyoles, A. Serenelli, F. L. Villante, S. Basu, J. Redondo, and J. Isern, New axion and hidden photon constraints from a solar data global fit, J. Cosmol. Astropart. Phys. 10 (2015) 015.

[14] J. H. Chang, R. Essig, and S. D. McDermott, Revisiting Supernova 1987A constraints on dark photons, J. High Energy Phys. 01 (2017) 107.

[15] E. Hardy and R. Lasenby, Stellar cooling bounds on new light particles: Plasma mixing effects, J. High Energy Phys. 02 (2017) 033.

[16] K. Ehret et al., New ALPS results on hidden-sector lightweights, Phys. Lett. B 689, 149 (2010).

[17] R. Bahre et al., Any light particle search II, Technical design report, J. Instrum. 8, T09001 (2013).

[18] OSQAR Collaboration, First results from the OSQAR photon regeneration experiment: No light shining through a wall, Phys. Rev. D 78, 092003 (2008).

[19] J. Kumar and J. D. Wells, CERN LHC and ILC probes of hidden-sector gauge bosons, Phys. Rev. D 74, 115017 (2006).

[20] D. Feldman, Z. Liu, and P. Nath, The Stueckelberg Z prime at the LHC: Discovery potential, signature spaces and model discrimination, J. High Energy Phys. 11 (2006) 007.

[21] J. Bramante, R. S. Hundi, J. Kumar, A. Rajaraman, and D. Yaylali, Collider searches for Fermiophobic gauge bosons, Phys. Rev. D 84, 115018 (2011).

[22] D. Curtin, R. Essig, S. Gori, and J. Shelton, Illuminating dark photons with high-energy colliders, J. High Energy Phys. 02 (2015) 157.

[23] D. Horns, J. Jaeckel, A. Lindner, A. Lobanov, J. Redondo, and A. Ringwald, Searching for WISPy cold dark matter with a dish antenna, J. Cosmol. Astropart. Phys. 04 (2013) 016. 
[24] J. Jaeckel and J. Redondo, Resonant to broadband searches for cold dark matter consisting of weakly interacting slim particles, Phys. Rev. D 88, 115002 (2013).

[25] MADMAX Working Group, Dielectric Haloscopes: A New Way to Detect Axion Dark Matter, Phys. Rev. Lett. 118, 091801 (2017).

[26] A. Arvanitaki, S. Dimopoulos, and K. Van Tilburg, Resonant Absorption of Bosonic Dark Matter in Molecules, Phys. Rev. X 8, 041001 (2018).

[27] M. Baryakhtar, J. Huang, and R. Lasenby, Axion and hidden photon dark matter detection with multilayer optical haloscopes, Phys. Rev. D 98, 035006 (2018).

[28] M. R. Baldeschi, G. B. Gelmini, and R. Ruffini, On massive fermions and bosons in galactic halos, Phys. Lett. B 122, 221 (1983).

[29] A. E. Nelson and J. Scholtz, Dark light, dark matter and the misalignment mechanism, Phys. Rev. D 84, 103501 (2011).

[30] P. Arias, D. Cadamuro, M. Goodsell, J. Jaeckel, J. Redondo, and A. Ringwald, WISPy cold dark matter, J. Cosmol. Astropart. Phys. 06 (2012) 013.

[31] P. W. Graham, J. Mardon, and S. Rajendran, Vector dark matter from inflationary fluctuations, Phys. Rev. D 93, 103520 (2016).

[32] J. A. Dror, K. Harigaya, and V. Narayan, Parametric resonance production of ultralight vector dark matter, Phys. Rev. D 99, 035036 (2019).

[33] P. Agrawal, N. Kitajima, M. Reece, T. Sekiguchi, and F. Takahashi, Relic abundance of dark photon dark matter, Phys. Lett. B 801, 135136 (2020).

[34] R. T. Co, A. Pierce, Z. Zhang, and Y. Zhao, Dark photon dark matter produced by axion oscillations, Phys. Rev. D 99, 075002 (2019).

[35] A. J. Long and L.-T. Wang, Dark photon dark matter from a network of cosmic strings, Phys. Rev. D 99, 063529 (2019).

[36] Y. Miyamoto, H. Hara, T. Masuda, N. Sasao, M. Tanaka, S. Uetake, A. Yoshimi, K. Yoshimura, and M. Yoshimura, Externally triggered coherent two-photon emission from hydrogen molecules, Prog. Theor. Exp. Phys. 2015, $081 \mathrm{C} 01$ (2015).

[37] T. Hiraki, H. Hara, Y. Miyamoto, K. Imamura, T. Masuda, N. Sasao, S. Uetake, A. Yoshimi, K. Yoshimura, and M. Yoshimura, Coherent two-photon emission from hydrogen molecules excited by counter-propagating laser pulses, J. Phys. B 52, 045401 (2019).

[38] M. Yoshimura, Neutrino pair emission from excited atoms, Phys. Rev. D 75, 113007 (2007).

[39] M. Yoshimura, C. Ohae, A. Fukumi, K. Nakajima, I. Nakano, H. Nanjo et al., Macro-coherent two photon and radiative neutrino pair emission, arXiv:0805.1970.

[40] A. Fukumi et al., Neutrino spectroscopy with atoms and molecules, Prog. Theor. Exp. Phys. 2012, 04 D002 (2012).

[41] M. Yoshimura, N. Sasao, and M. Tanaka, Dynamics of paired superradiance, Phys. Rev. A 86, 013812 (2012).

[42] Y. Miyamoto, H. Hara, S. Kuma, T. Masuda, I. Nakano, C. Ohae et al., Observation of coherent two-photon emission from the first vibrationally excited state of hydrogen molecules, Prog. Theor. Exp. Phys. 2014, 113 C01 (2014).

[43] H. Hara, Y. Miyamoto, T. Hiraki, T. Masuda, N. Sasao, S. Uetake, A. Yoshimi, K. Yoshimura, and M. Yoshimura, Frequency dependence of coherently amplified two-photon emission from hydrogen molecules, Phys. Rev. A 96, 063827 (2017).

[44] Y. Miyamoto, H. Hara, T. Masuda, N. Sasao, S. Uetake, A. Yoshimi, K. Yoshimura, and M. Yoshimura, Vibrational two-photon emission from coherently excited solid parahydrogen, J. Phys. Chem. A 121, 3943 (2017).

[45] N. Song, R. Boyero Garcia, J. J. Gomez-Cadenas, M. C. Gonzalez-Garcia, A. Peralta Conde, and J. Taron, Conditions for statistical determination of the neutrino mass spectrum in radiative emission of neutrino Pairs in atoms, Phys. Rev. D 93, 013020 (2016).

[46] R. Boyero Garcia, A. V. Carpentier, J. J. Gomez-Cadenas, and A. Peralta Conde, A novel technique to achieve atomic macro-coherence as a tool to determine the nature of ..., Appl. Phys. B 122, 262 (2016).

[47] J. M. Vaquero, J. Cuevas-Maraver, and A. P. Conde, Propagation studies for the construction of atomic macrocoherence in dense media as a tool to investigate neutrino physics, Eur. Phys. J. D 71, 61 (2017).

[48] J. Zhang and S. Zhou, Improved statistical determination of absolute neutrino masses via radiative emission of neutrino pairs from atoms, Phys. Rev. D 93, 113020 (2016).

[49] N. Sasao and M. Yoshimura, New method of galactic axion search, Eur. Phys. J. C 78, 949 (2018).

[50] G.-Y. Huang and S. Zhou, Probing cosmic Aaxions through resonant emission and absorption in atomic systems with superradiance, Phys. Rev. D 100, 035010 (2019).

[51] C. Alvarez-Luna and J. A. R. Cembranos, Dark photon searches with atomic transitions, J. High Energy Phys. 07 (2019) 110.

[52] V. V. Flambaum, I. B. Samsonov, and H. B. Tran Tan, Interference-assisted detection of dark photon using atomic transitions, Phys. Rev. D 99, 115019 (2019).

[53] P. Sikivie, Axion Dark Matter Detection Using Atomic Transitions, Phys. Rev. Lett. 113, 201301 (2014).

[54] R. H. Dicke, Coherence in spontaneous radiation processes, Phys. Rev. 93, 99 (1954).

[55] L. M. Narducci, W. W. Eidson, P. Furcinitti, and D. C. Eteson, Theory of a two-photon laser amplifier, Phys. Rev. A 16, 1665 (1977).

[56] S. E. Harris and M. Jain, Optical parametric oscillators pumped by population-trapped atoms, Opt. Lett. 22, 636 (1997).

[57] C.-Y. Kuo, R. J. Kerl, N. D. Patel, and C. K. N. Patel, Nonradiative Relaxation of Excited Vibrational States of Solid Hydrogen, Phys. Rev. Lett. 53, 2575 (1984).

[58] J. Li, M. Suzuki, M. Katsuragawa, and K. Hakuta, Measurement of the energy decay rate for the first vibrationalexcited-state in solid parahydrogen, J. Chem. Phys. 115, 930 (2001).

[59] C. Delalande and G. Gale, Low temperature vibrational relaxation of nh2 gas, liquid and solid, Chem. Phys. Lett. 50, 339 (1977).

[60] W. K. Bischel and M. J. Dyer, Temperature dependence of the raman linewidth and line shift for the $q(1)$ and $q(0)$ transitions in normal and para- $\mathrm{h}_{2}$, Phys. Rev. A 33, 3113 (1986).

[61] EKSPLA Nd:YAG Laser Link.

[62] O. Schwartz, J. J. Axelrod, P. Haslinger, C. Ophus, R. M. Glaeser, and H. Müller, Continuous $40 \mathrm{GW} / \mathrm{cm}^{2}$ laser 
intensity in a near-concentric optical cavity, Opt. Express 25, 14453 (2017).

[63] T. Momose and T. Oka, High-resolution stimulated raman gain spectroscopy of parahydrogen crystals, J. Low Temp. Phys. 139, 515 (2005).

[64] M. Ahlers, H. Gies, J. Jaeckel, J. Redondo, and A. Ringwald, Light from the hidden sector, Phys. Rev. D 76, 115005 (2007).

[65] M. Ahlers, H. Gies, J. Jaeckel, J. Redondo, and A. Ringwald, Laser experiments explore the hidden sector, Phys. Rev. D 77, 095001 (2008).

[66] J. Redondo, Atlas of solar hidden photon emission, J. Cosmol. Astropart. Phys. 07 (2015) 024.

[67] J. Jaeckel and S. Roy, Spectroscopy as a test of Coulomb's law: A probe of the hidden sector, Phys. Rev. D 82, 125020 (2010).

[68] E. R. Williams, J. E. Faller, and H. A. Hill, New Experimental Test of Coulomb's Law: A Laboratory Upper
Limit on the Photon Rest Mass, Phys. Rev. Lett. 26, 721 (1971).

[69] J. Jaeckel, J. Redondo, and A. Ringwald, Signatures of a Hidden Cosmic Microwave Background, Phys. Rev. Lett. 101, 131801 (2008).

[70] A. Mirizzi, J. Redondo, and G. Sigl, Microwave background constraints on mixing of photons with hidden photons, J. Cosmol. Astropart. Phys. 03 (2009) 026.

[71] M. Betz, F. Caspers, M. Gasior, M. Thumm, and S. W. Rieger, First results of the CERN resonant weakly interacting sub-eV particle search (CROWS), Phys. Rev. D 88, 075014 (2013).

[72] J.H. Steffen and A. Upadhye, The GammeV suite of experimental searches for axion-like particles, Mod. Phys. Lett. A 24, 2053 (2009).

[73] M. Yoshimura, Light propagation and paired superradiance in coherent medium, Prog. Theor. Phys. 125, 149 (2011). 